Supporting Information for:

\title{
Cu-Catalyzed Pyridine Synthesis via Oxidative Annulation of Cyclic Ketones with Propargyl Amine
}

Svitlana O. Sotnik, ${ }^{a}$ Andrii I. Subota, ${ }^{\text {a,b }}$ Anton Y. Kliuchynskyi, ${ }^{\mathrm{c}}$ Dmytro V. Yehorov, ${ }^{\mathrm{a}, \mathrm{b}}$

Anton S. Lytvynenko, ${ }^{d}$ Alexander B. Rozhenko, ${ }^{\text {be }}$ Sergey V. Kolotilov, ${ }^{d}$

Sergey V. Ryabukhin, ${ }^{\mathrm{a}, \mathrm{c}^{*}}$ Dmitriy M. Volochnyuk ${ }^{\mathrm{a}, \mathrm{b}, \mathrm{c}^{*}}$

aEnamine Ltd, 78 Chervonotkatska str., Kyiv, 02094, Ukraine

${ }^{b}$ Institute of Organic Chemistry, National Academy of Sciences of Ukraine, 5 Murmanska str., Kyiv, 02094, Ukraine

"National Taras Shevchenko University of Kyiv, 60 Volodymyrska str., Kyiv, 01033, Ukraine

${ }^{\mathrm{d}}$ L.V. Pisarzhevskii Institute of Physical Chemistry of the National Academy of Sciences of

Ukraine, 31 Nauki ave., Kyiv, 03028, Ukraine

"National Technical University of Ukraine "Igor Sikorsky Kyiv Polytechnic Institute”, 37

Peremogy ave., Kyiv, 03056, Ukraine

E-mail: s.v.ryabukhin@gmail.com. Phone:+380506424763;

d.volochnyuk@gmail.com. Phone:+380967139494.

Table of contents

5,6,7,8-tetrahydroquinoline (23a), ${ }^{1} \mathrm{H}$ NMR (400 MHz, $\mathrm{CDCl}_{3}$ ) .3

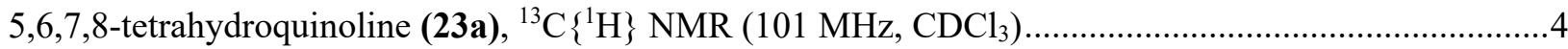

5H,6H,7H-cyclopenta[b]pyridine (23b), ${ }^{1} \mathrm{H}$ NMR (400 MHz, $\left.\mathrm{CDCl}_{3}\right)$...............................................5

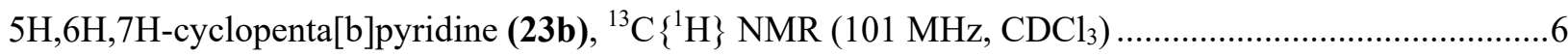

Ethyl 5,6,7,8-tetrahydroquinoline-6-carboxylate (23c), ${ }^{1} \mathrm{H}$ NMR $\left(400 \mathrm{MHz}, \mathrm{CDCl}_{3}\right)$.............................

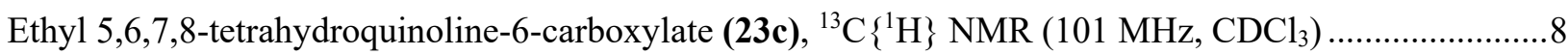

5H,6H,7H,8H,9H-cyclohepta[b]pyridine (23d), ${ }^{1} \mathrm{H}$ NMR (400 MHz, $\left.\mathrm{CDCl}_{3}\right)$......................................

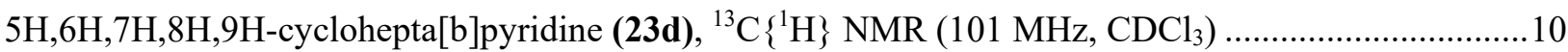

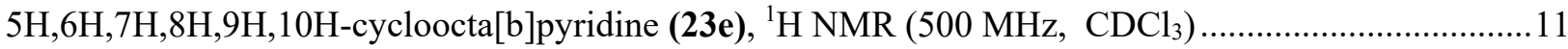

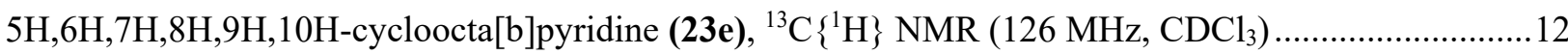

Mixture of ethyl 5,6,7,8-tetrahydroquinoline-5-carboxylate and ethyl 5,6,7,8-tetrahydroquinoline-7carboxylate (23g), ${ }^{1} \mathrm{H}$ NMR $\left(400 \mathrm{MHz}, \mathrm{CDCl}_{3}\right)$.

Mixture of ethyl 5,6,7,8-tetrahydroquinoline-5-carboxylate and ethyl 5,6,7,8-tetrahydroquinoline-7-

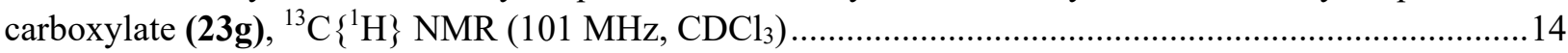

2H,3H-1 $\lambda^{6}$-thieno[3,2-b]pyridine-1,1-dione (23k), ${ }^{1} \mathrm{H}$ NMR (400 MHz, $\mathrm{CDCl}_{3}$ ) ...................................15

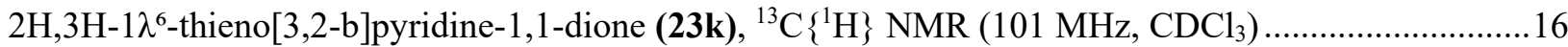

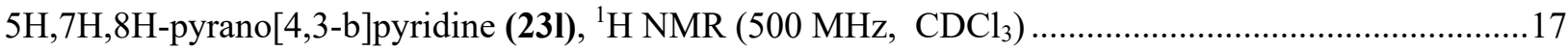

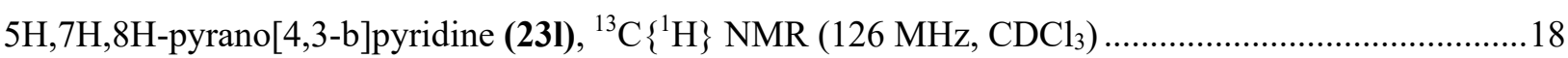

Tert-butyl 5,6,7,8-tetrahydro-1,6-naphthyridine-6-carboxylate (23m), ${ }^{1} \mathrm{H}$ NMR (400 MHz, $\mathrm{CDCl}_{3}$ ) ......19

Tert-butyl 5,6,7,8-tetrahydro-1,6-naphthyridine-6-carboxylate (23m), ${ }^{13} \mathrm{C}\left\{{ }^{1} \mathrm{H}\right\}$ NMR $\left(126 \mathrm{MHz}, \mathrm{CDCl}_{3}\right)$

Tert-butyl 1,2,3,4-tetrahydro-1,5-naphthyridine-1-carboxylate (23p), ${ }^{1} \mathrm{H}$ NMR (400 MHz, $\left.\mathrm{CDCl}_{3}\right)$.......21 
Tert-butyl 1,2,3,4-tetrahydro-1,5-naphthyridine-1-carboxylate (23p), ${ }^{13} \mathrm{C}\left\{{ }^{1} \mathrm{H}\right\}$ NMR (101 MHz, CDCl $) 22$

Tert-butyl N-(5,6,7,8-tetrahydroquinolin-6-yl)carbamate (23q), ${ }^{1} \mathrm{H}$ NMR $\left(400 \mathrm{MHz}, \mathrm{CDCl}_{3}\right) \ldots \ldots \ldots \ldots \ldots . . .23$

Tert-butyl N-(5,6,7,8-tetrahydroquinolin-6-yl)carbamate (23q), ${ }^{13} \mathrm{C}\left\{{ }^{1} \mathrm{H}\right\}$ NMR $\left(101 \mathrm{MHz}, \mathrm{CDCl}_{3}\right) \ldots \ldots . .24$

7',8'-dihydro-5'H-spiro[1,3-dioxolane-2,6'-quinoline] (23r), ${ }^{1} \mathrm{H}$ NMR (500 MHz, $\left.\mathrm{CDCl}_{3}\right)$....................25

7', 8'-dihydro-5'H-spiro[1,3-dioxolane-2,6'-quinoline] (23r), ${ }^{13} \mathrm{C}\left\{{ }^{1} \mathrm{H}\right\}$ NMR (126 MHz, $\left.\mathrm{CDCl}_{3}\right)$.............26

5,6,7,8-tetrahydroquinolin-5-one (23u), ${ }^{1} \mathrm{H}$ NMR (500 MHz, DMSO-d $\left.\mathrm{d}_{6}\right)$.............................................2

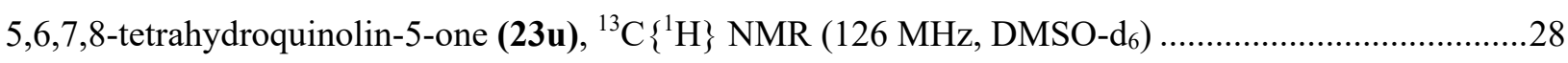

5,6,7,8-tetrahydroquinoline-6-carboxylic acid (24), ${ }^{1} \mathrm{H}$ NMR (400 MHz, DMSO-d 6 ) ...........................29

5,6,7,8-tetrahydroquinoline-6-carboxylic acid (24), ${ }^{13} \mathrm{C}\left\{{ }^{1} \mathrm{H}\right\}$ NMR (151 MHz, DMSO-d 6 ) .....................30

5,6,7,8-tetrahydro-1,6-naphthyridine-1,6-diium chloride (25), ${ }^{1} \mathrm{H}$ NMR (400 MHz, DMSO-d $\left.\mathrm{d}_{6}\right) \ldots \ldots \ldots \ldots . . . . .31$

5,6,7,8-tetrahydro-1,6-naphthyridine-1,6-diium chloride (25), ${ }^{13} \mathrm{C}\left\{{ }^{1} \mathrm{H}\right\}$ NMR (151 MHz, DMSO-d 6 ) .....32

1,2,3,4-tetrahydro-1,5-naphthyridine (26), ${ }^{1} \mathrm{H}$ NMR $\left(400 \mathrm{MHz}, \mathrm{CDCl}_{3}\right)$..............................................33

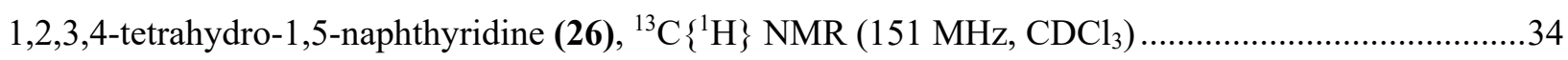

5,6,7,8-tetrahydroquinolin-6-aminium chloride (27), ${ }^{1} \mathrm{H}$ NMR (400 MHz, DMSO-d $\mathrm{d}_{6}$ ) ............................35

5,6,7,8-tetrahydroquinolin-6-aminium chloride (27), ${ }^{13} \mathrm{C}\left\{{ }^{1} \mathrm{H}\right\}$ NMR (101 MHz, DMSO-d $\left.\mathrm{d}_{6}\right) \ldots \ldots \ldots \ldots \ldots \ldots . . . . . . . .36$

5,6,7,8-tetrahydroquinolin-6-one (28), ${ }^{1} \mathrm{H}$ NMR $\left(400 \mathrm{MHz}, \mathrm{CDCl}_{3}\right)$....................................................37

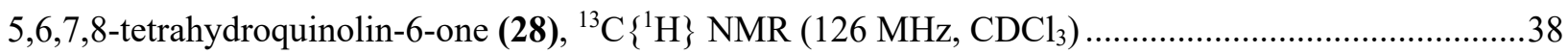


5,6,7,8-tetrahydroquinoline (23a), ${ }^{1} \mathrm{H} \mathrm{NMR}\left(400 \mathrm{MHz}, \mathrm{CDCl}_{3}\right)$

Y

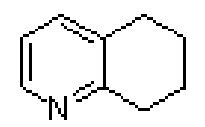

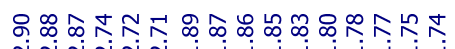

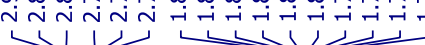
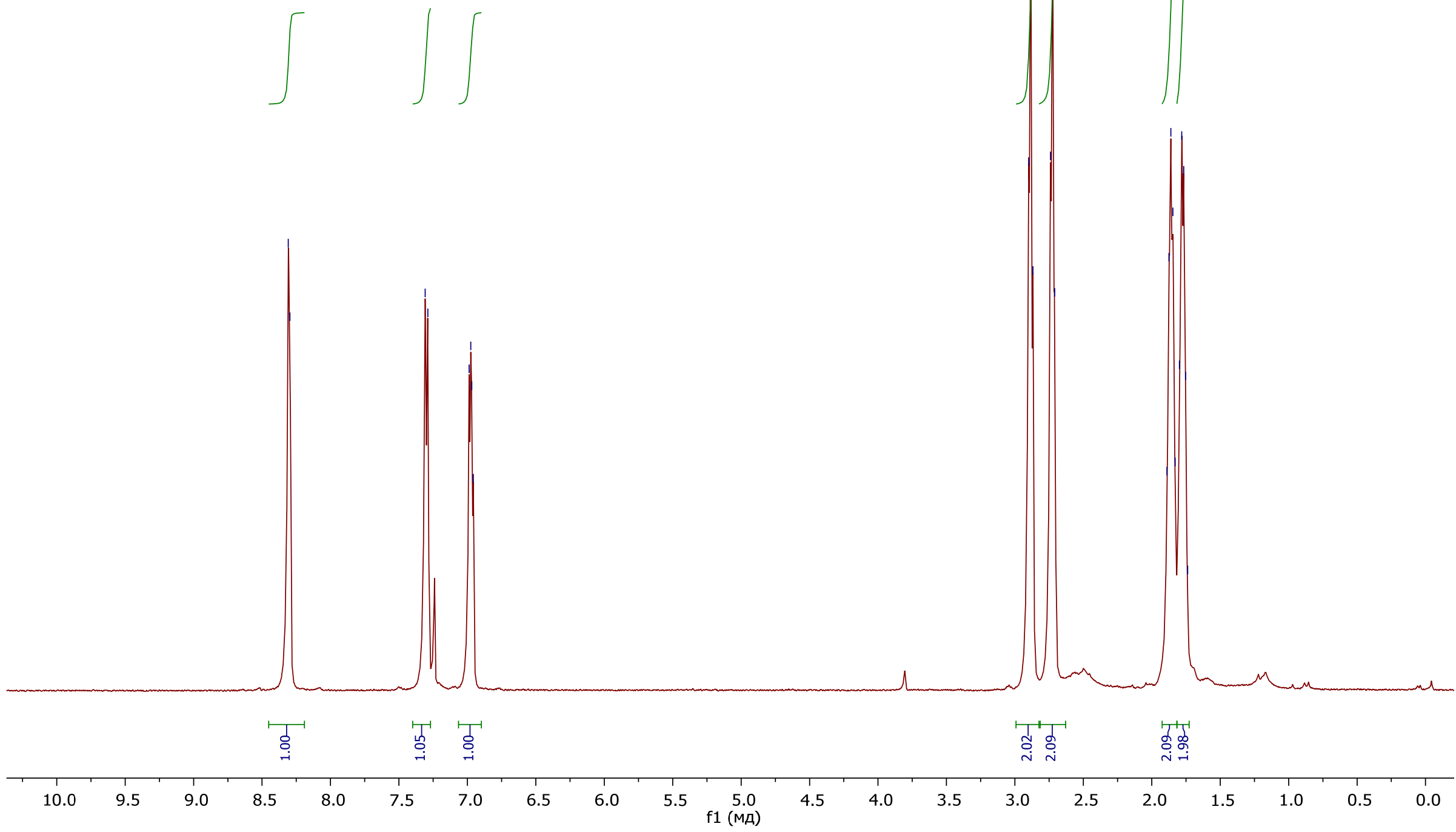
5,6,7,8-tetrahydroquinoline (23a), ${ }^{13} \mathrm{C}\left\{{ }^{1} \mathrm{H}\right\} \mathrm{NMR}\left(101 \mathrm{MHz}, \mathrm{CDCl}_{3}\right)$
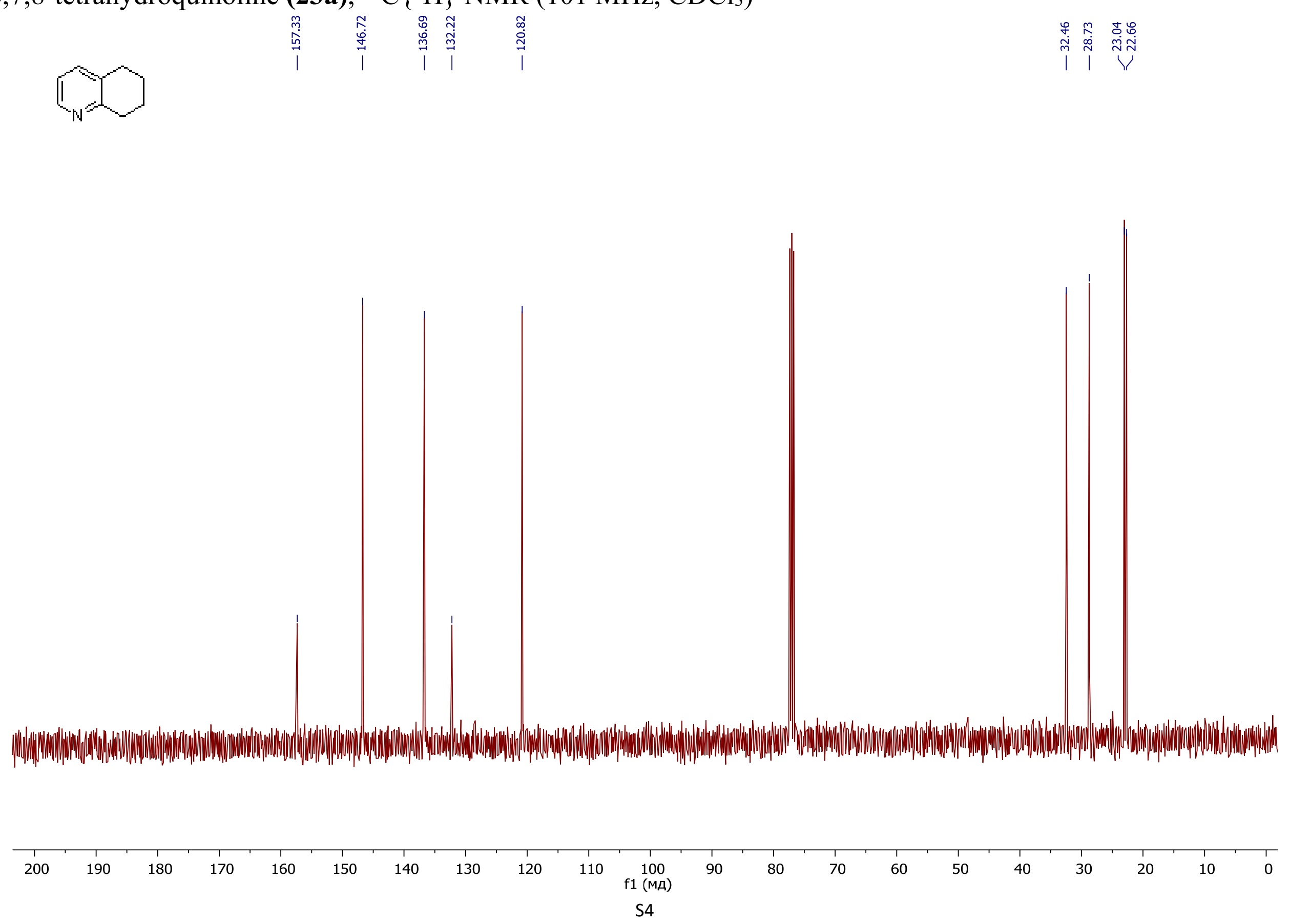
5H,6H,7H-cyclopenta[b]pyridine (23b), ${ }^{1} \mathrm{H} \mathrm{NMR}\left(400 \mathrm{MHz}, \mathrm{CDCl}_{3}\right.$ )

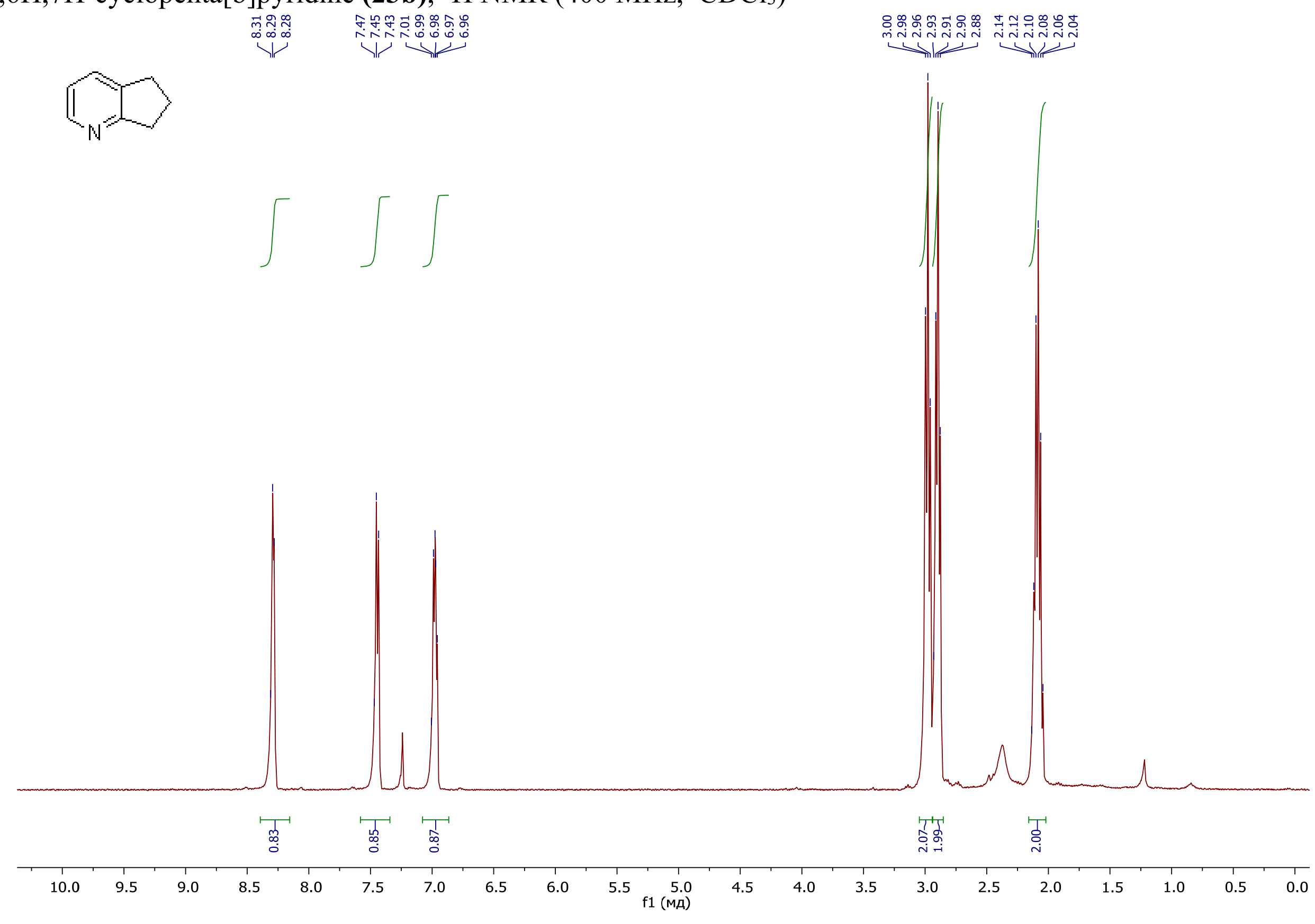


5H,6H,7H-cyclopenta[b]pyridine (23b), ${ }^{13} \mathrm{C}\left\{{ }^{1} \mathrm{H}\right\}$ NMR (101 MHz, $\left.\mathrm{CDCl}_{3}\right)$
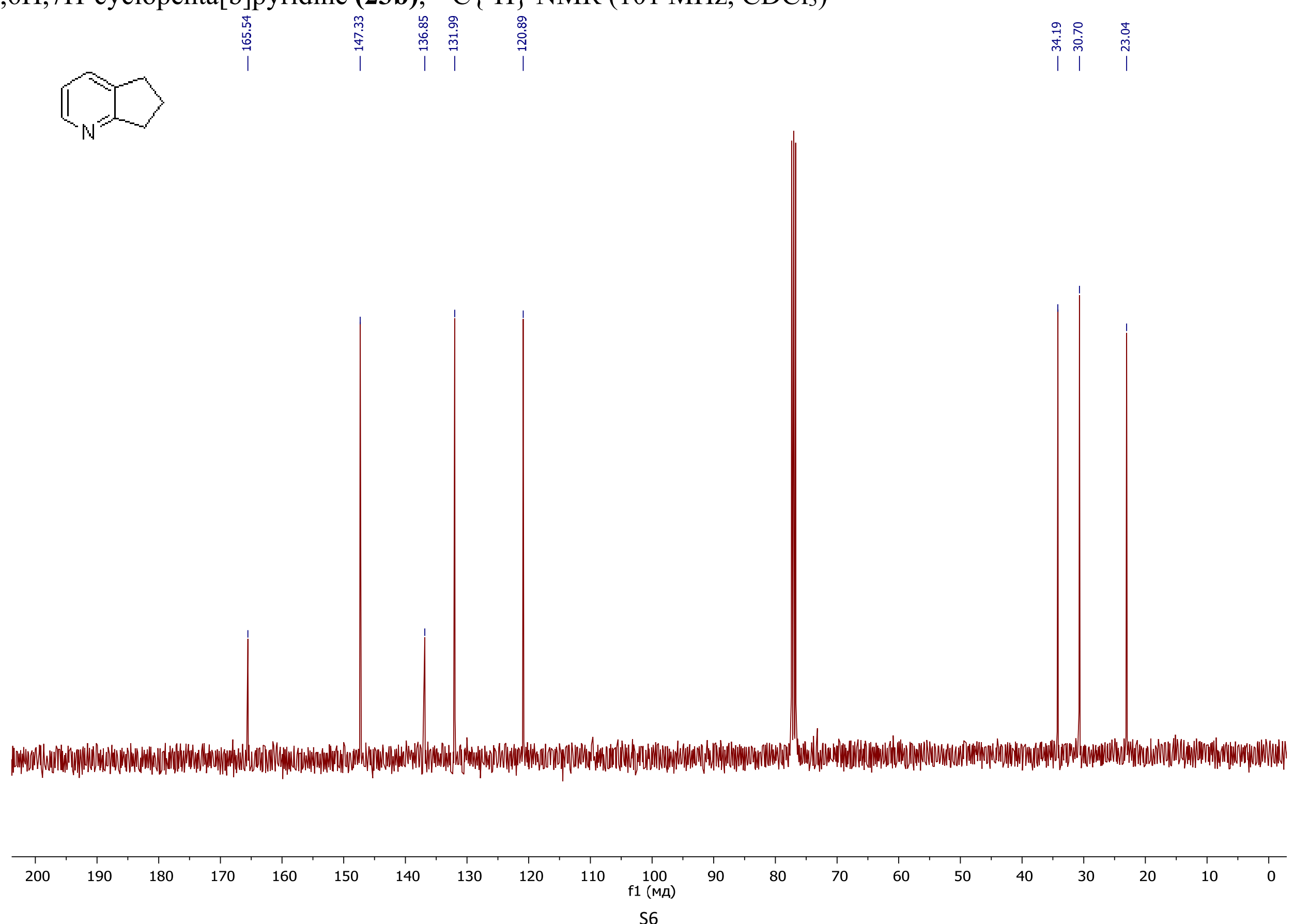
Ethyl 5,6,7,8-tetrahydroquinoline-6-carboxylate (23c), ${ }^{1} \mathrm{H}$ NMR (400 MHz, $\mathrm{CDCl}_{3}$ )

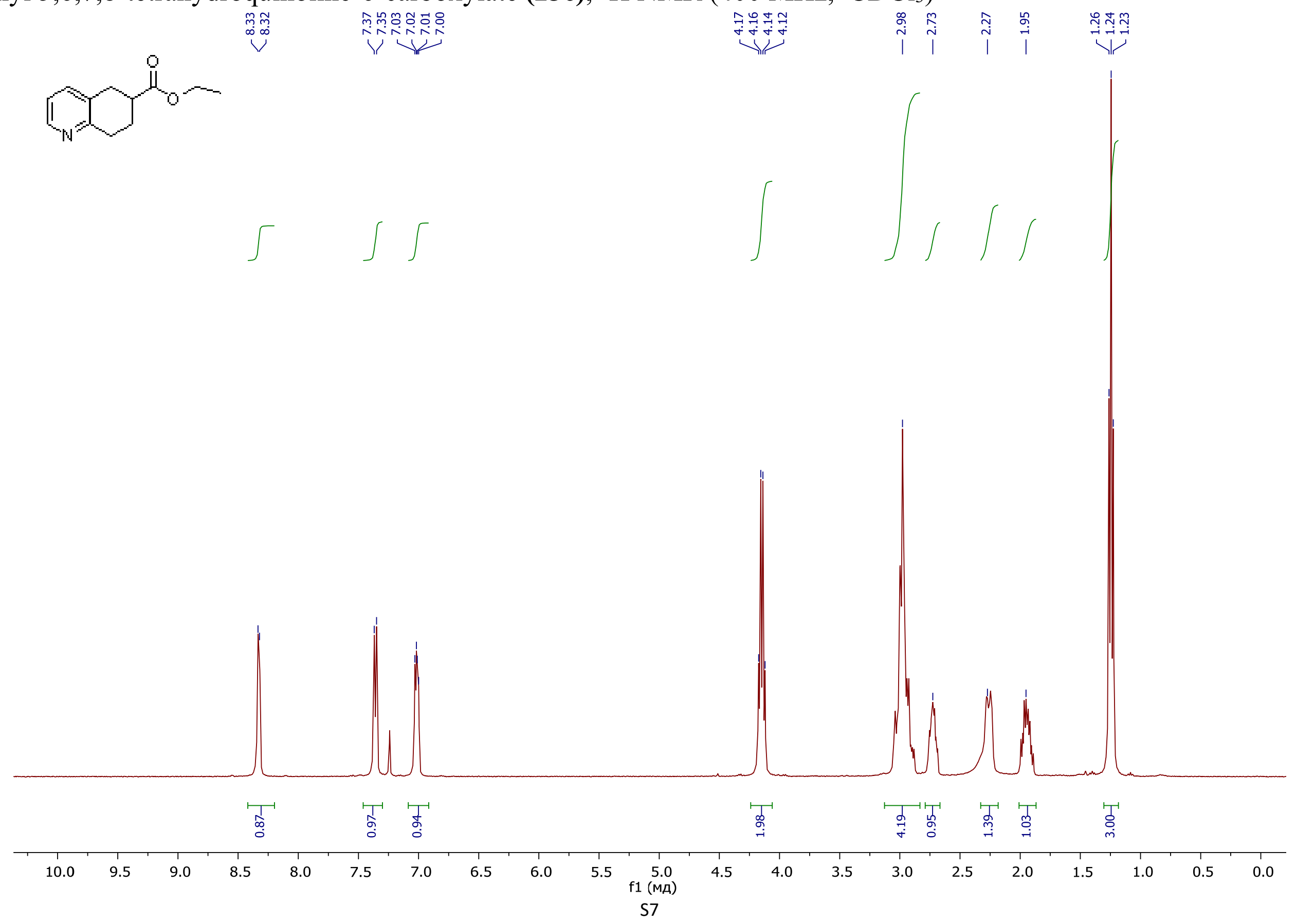


Ethyl 5,6,7,8-tetrahydroquinoline-6-carboxylate (23c), ${ }^{13} \mathrm{C}\left\{{ }^{1} \mathrm{H}\right\} \mathrm{NMR}\left(101 \mathrm{MHz}, \mathrm{CDCl}_{3}\right)$
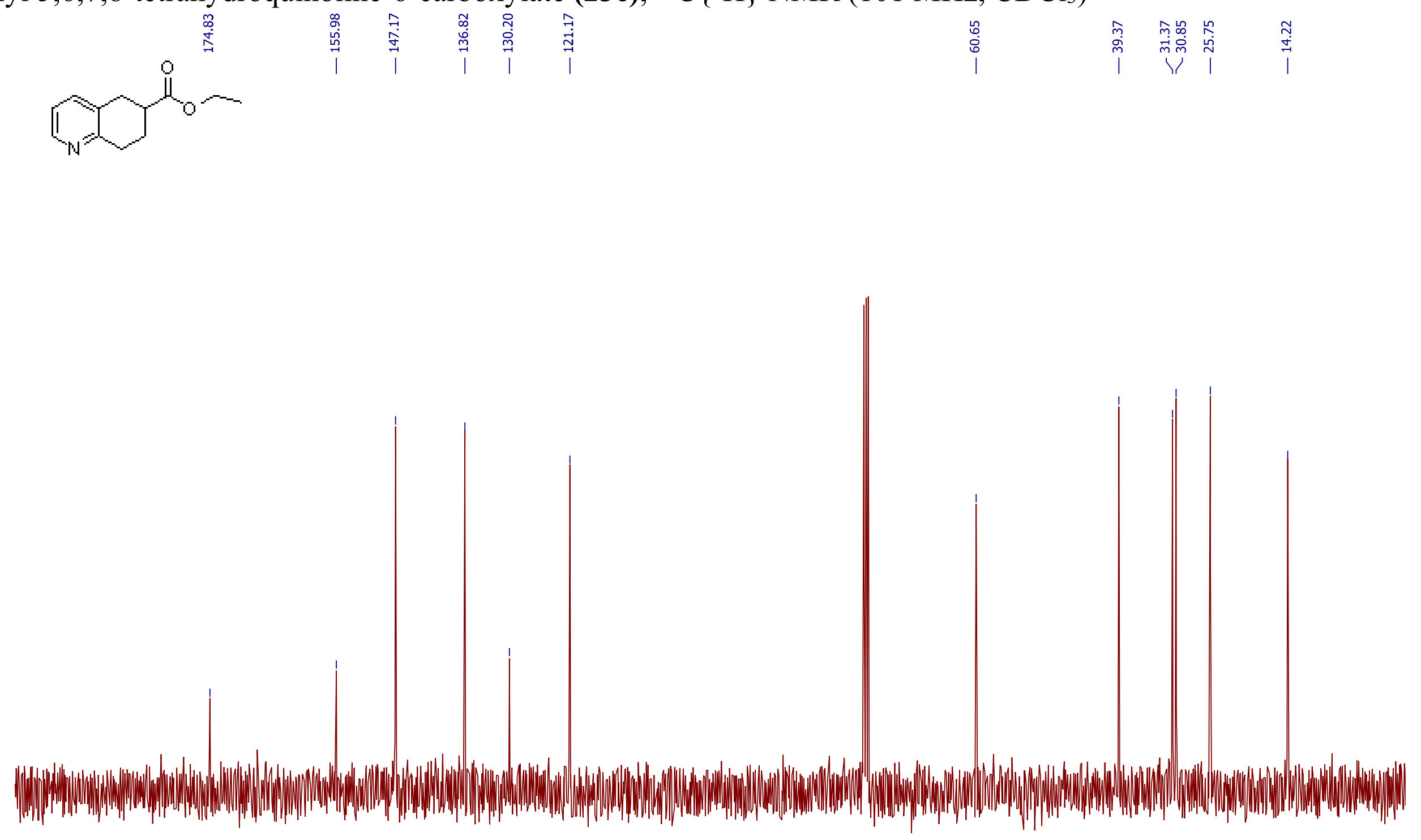

\begin{tabular}{|c|c|c|c|c|c|c|c|c|c|c|c|c|c|c|c|c|c|c|c|c|}
\hline 200 & 190 & 180 & 1 & 160 & 1150 & 140 & 130 & 120 & 110 & 100 & 1 & 80 & 70 & 60 & 1 & 40 & 30 & 20 & 10 & 0 \\
\hline 200 & 190 & 180 & 170 & 160 & 150 & 140 & 130 & 120 & 110 & $\begin{array}{c}100 \\
\mathrm{f} 1(\mathrm{MA})\end{array}$ & 90 & 80 & 70 & 60 & 50 & 40 & 30 & 20 & 10 & 0 \\
\hline
\end{tabular}


5H,6H,7H,8H,9H-cyclohepta[b]pyridine (23d), ${ }^{1} \mathrm{H}$ NMR (400 MHz, $\mathrm{CDCl}_{3}$ )

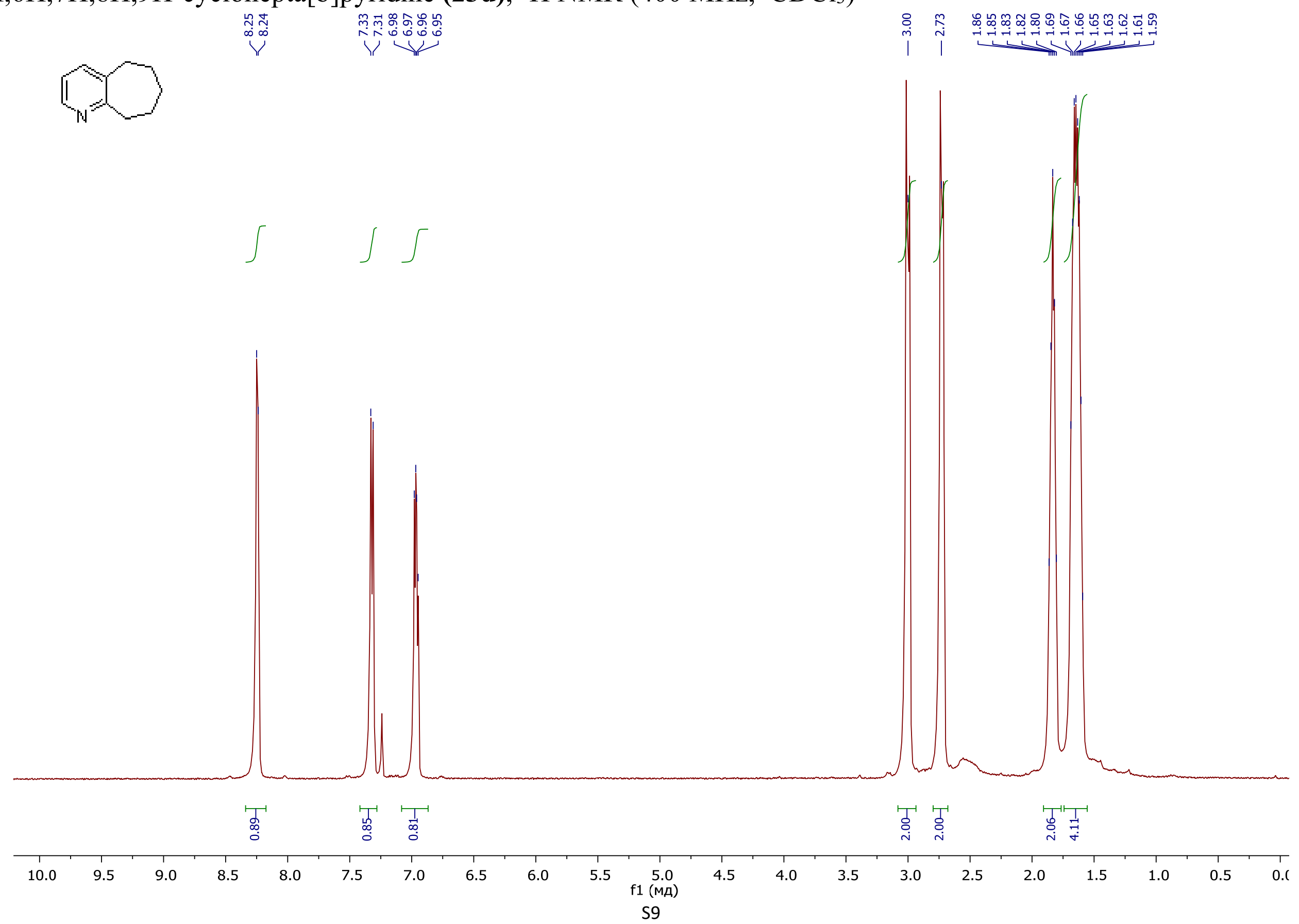


5H,6H,7H,8H,9H-cyclohepta[b]pyridine (23d), ${ }^{13} \mathrm{C}\left\{{ }^{1} \mathrm{H}\right\} \mathrm{NMR}\left(101 \mathrm{MHz}, \mathrm{CDCl}_{3}\right)$

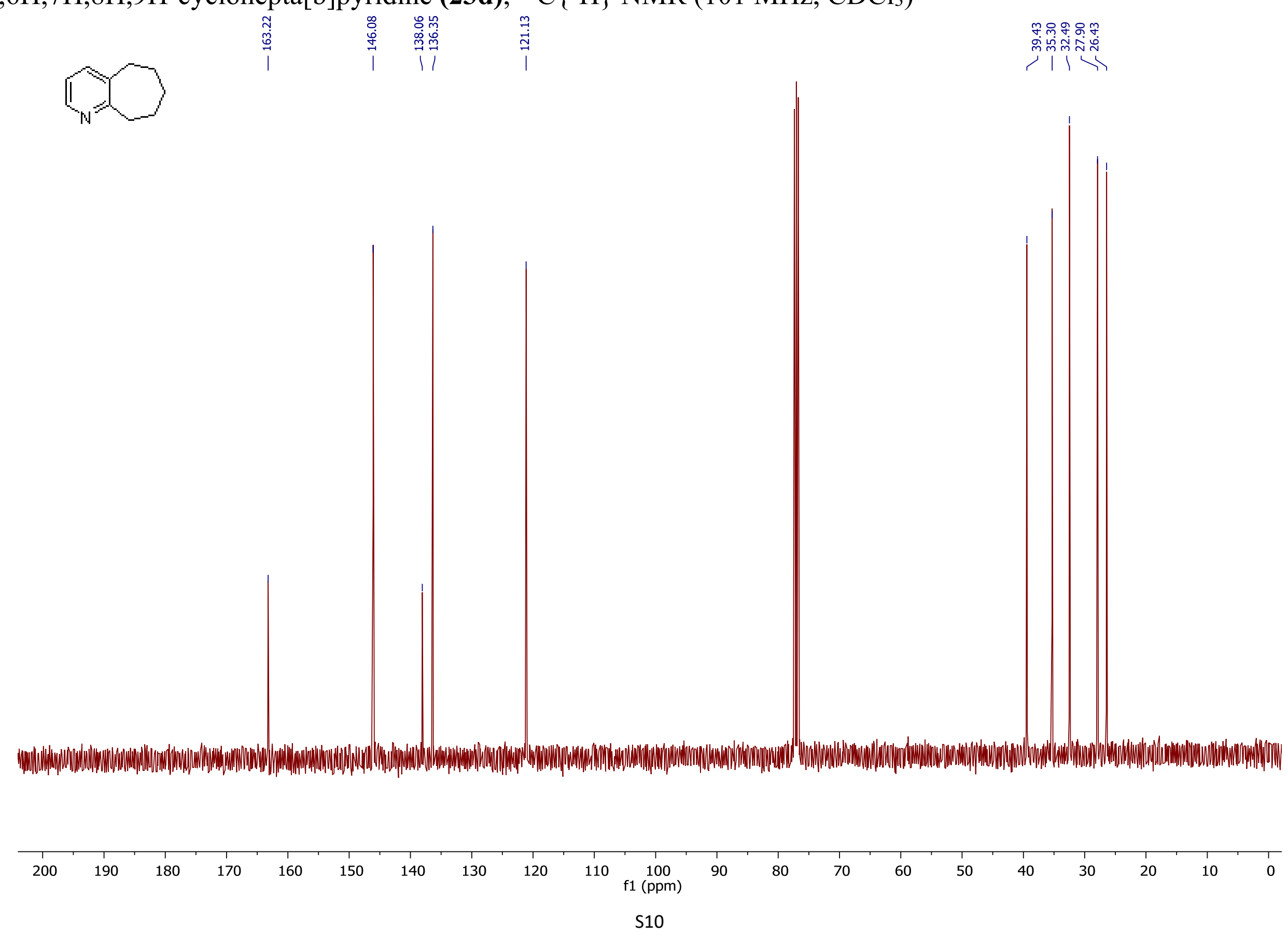


5H,6H,7H,8H,9H,10H-cycloocta[b]pyridine (23e), ${ }^{1} \mathrm{H}$ NMR (500 MHz, $\left.\mathrm{CDCl}_{3}\right)$

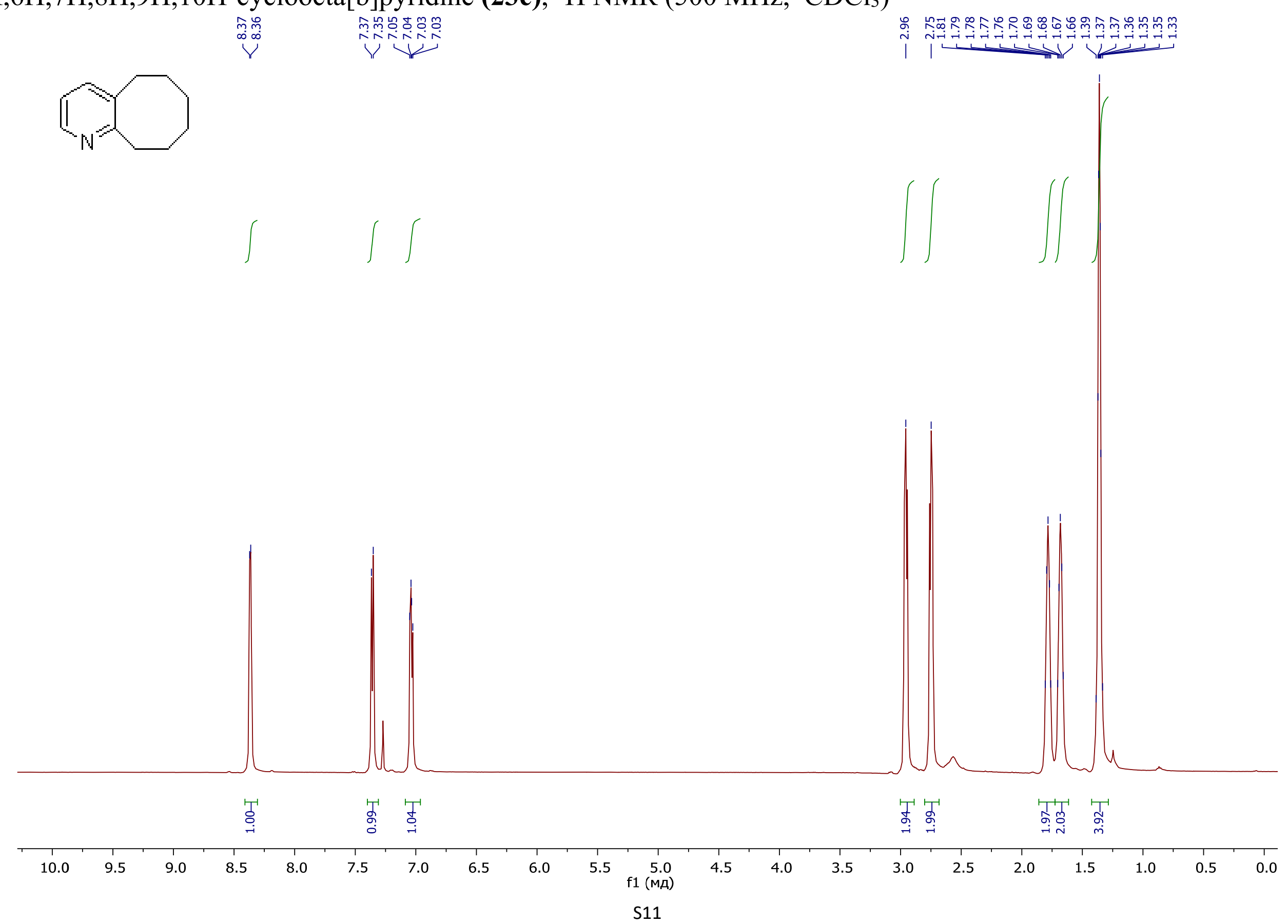


$5 \mathrm{H}, 6 \mathrm{H}, 7 \mathrm{H}, 8 \mathrm{H}, 9 \mathrm{H}, 10 \mathrm{H}$-cycloocta[b]pyridine (23e), ${ }^{13} \mathrm{C}\left\{{ }^{1} \mathrm{H}\right\} \mathrm{NMR}\left(126 \mathrm{MHz}, \mathrm{CDCl}_{3}\right)$

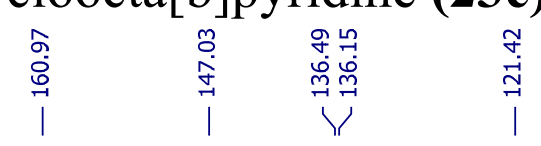

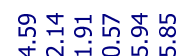

ले

事

(1)

$\backslash V\rangle$

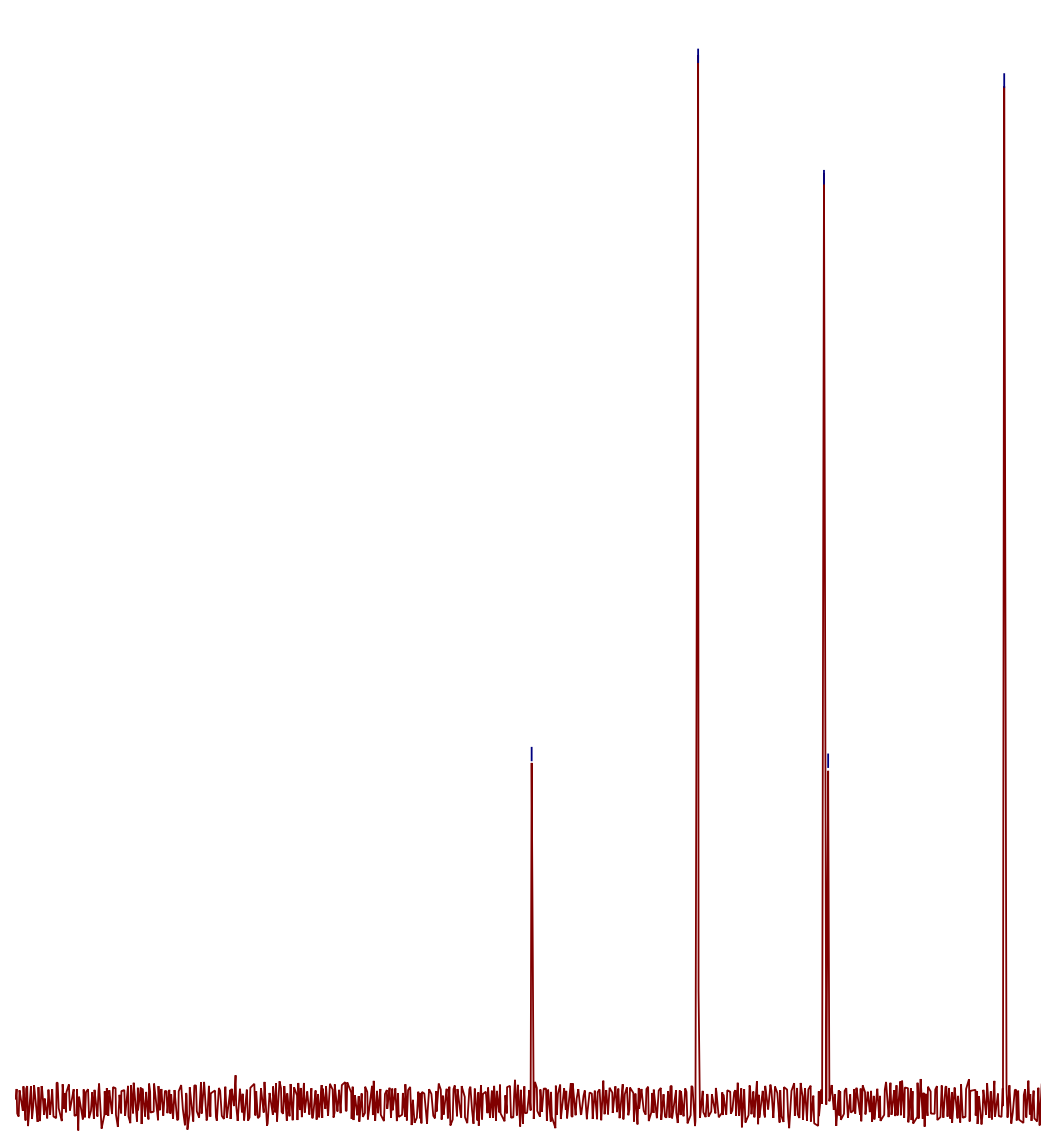

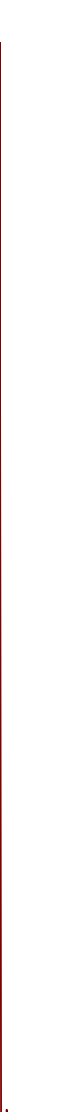

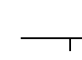


Mixture of ethyl 5,6,7,8-tetrahydroquinoline-5-carboxylate and ethyl 5,6,7,8-tetrahydroquinoline-7-carboxylate (23g), ${ }_{1}^{1} \mathrm{H}$ NMR $\left(400 \mathrm{MHz}, \mathrm{CDCl}_{3}\right)$

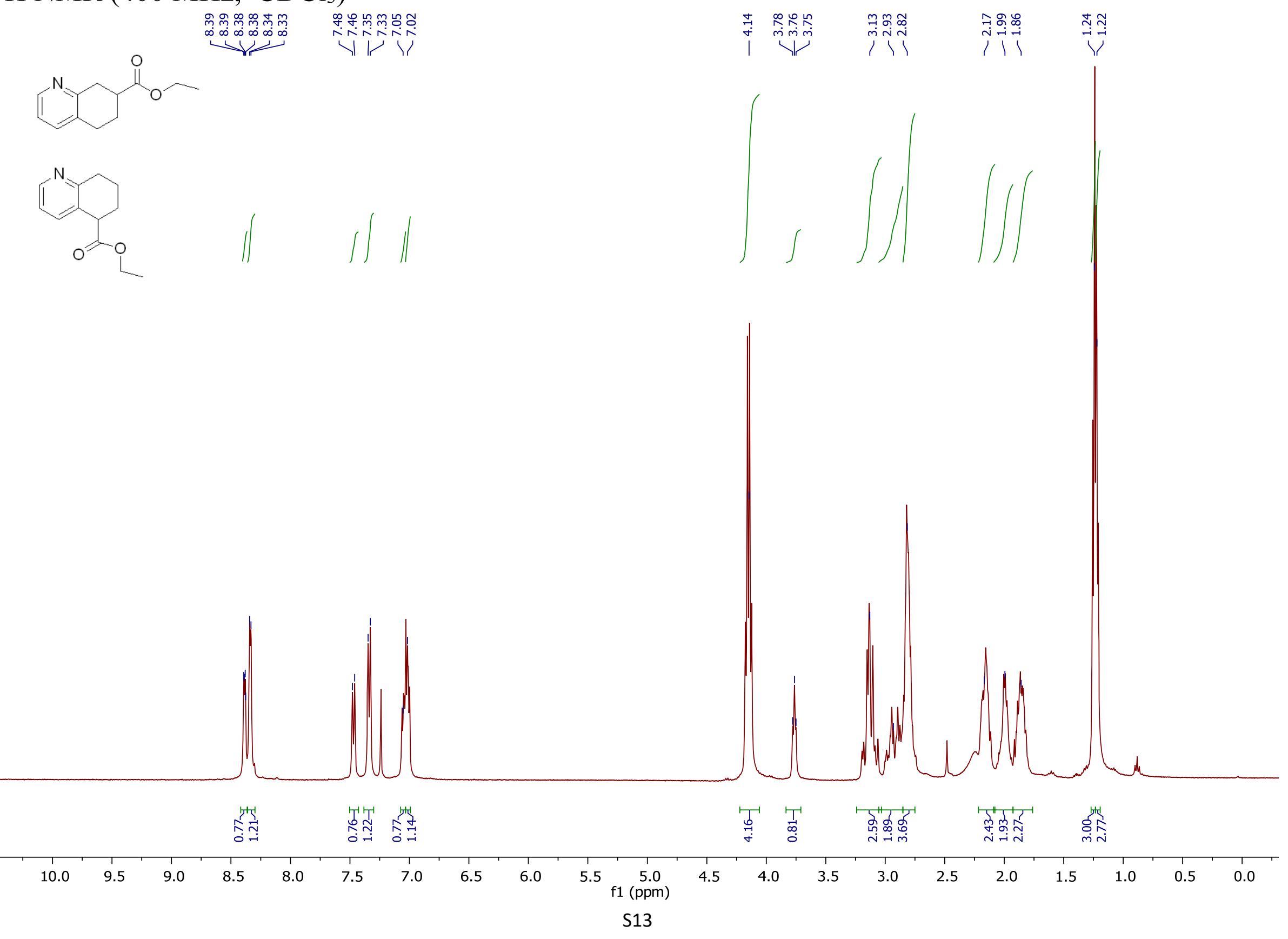


Mixture of ethyl 5,6,7,8-tetrahydroquinoline-5-carboxylate and ethyl 5,6,7,8-tetrahydroquinoline-7-carboxylate (23g), ${ }^{13} \mathrm{C}\left\{{ }^{1} \mathrm{H}\right\}$ NMR $\left(101 \mathrm{MHz}, \mathrm{CDCl}_{3}\right)$

ل/
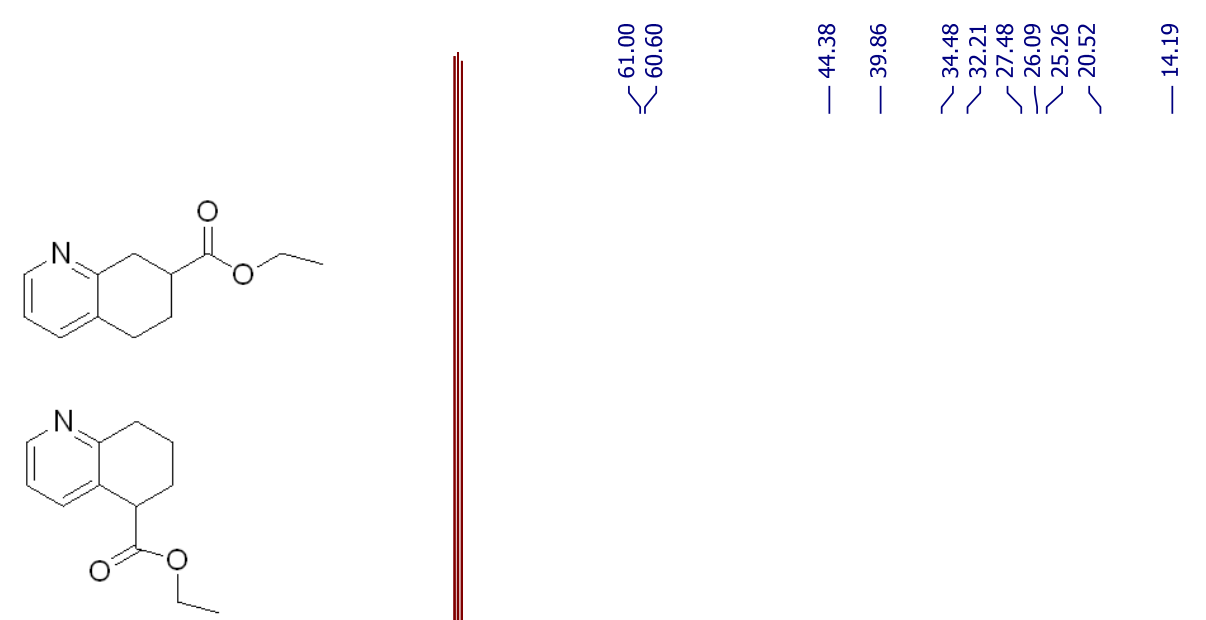

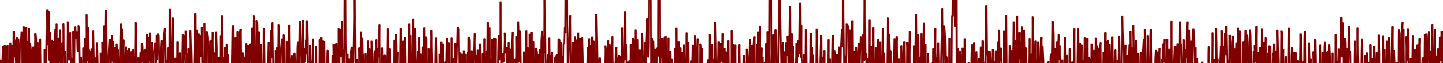

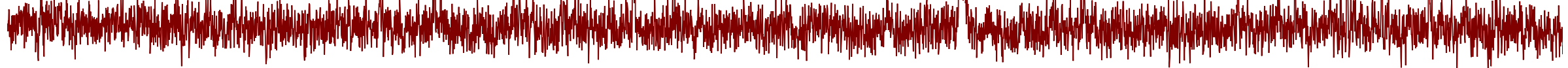

\begin{tabular}{|c|c|c|c|c|c|c|c|c|c|c|c|c|c|c|c|c|c|c|c|c|}
\hline 200 & 190 & 180 & 170 & 160 & 150 & 140 & 130 & 120 & 110 & $\begin{array}{c}100 \\
\mathrm{f} 1(\mathrm{ppm})\end{array}$ & 90 & 80 & 70 & 60 & 50 & 40 & 30 & 20 & 10 & 0 \\
\hline
\end{tabular}


2H,3H-1 $\lambda^{6}$-thieno[3,2-b]pyridine-1,1-dione (23k), ${ }^{1} \mathrm{H}$ NMR $\left(400 \mathrm{MHz}, \mathrm{CDCl}_{3}\right)$

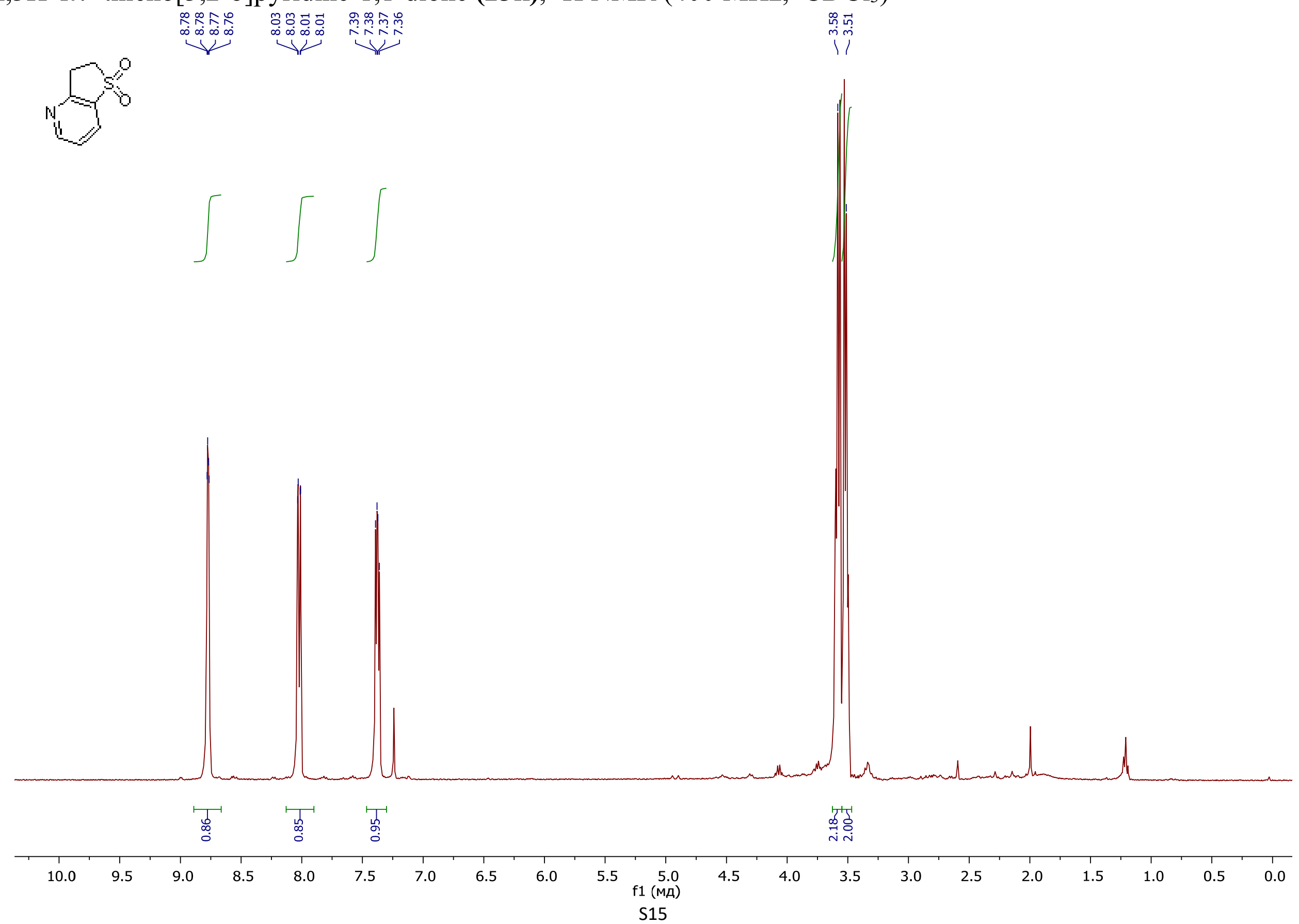


2H,3H-1 $\lambda^{6}$-thieno[3,2-b]pyridine-1,1-dione (23k), ${ }^{13} \mathrm{C}\left\{{ }^{1} \mathrm{H}\right\} \mathrm{NMR}\left(101 \mathrm{MHz}, \mathrm{CDCl}_{3}\right)$

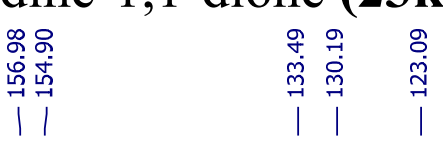

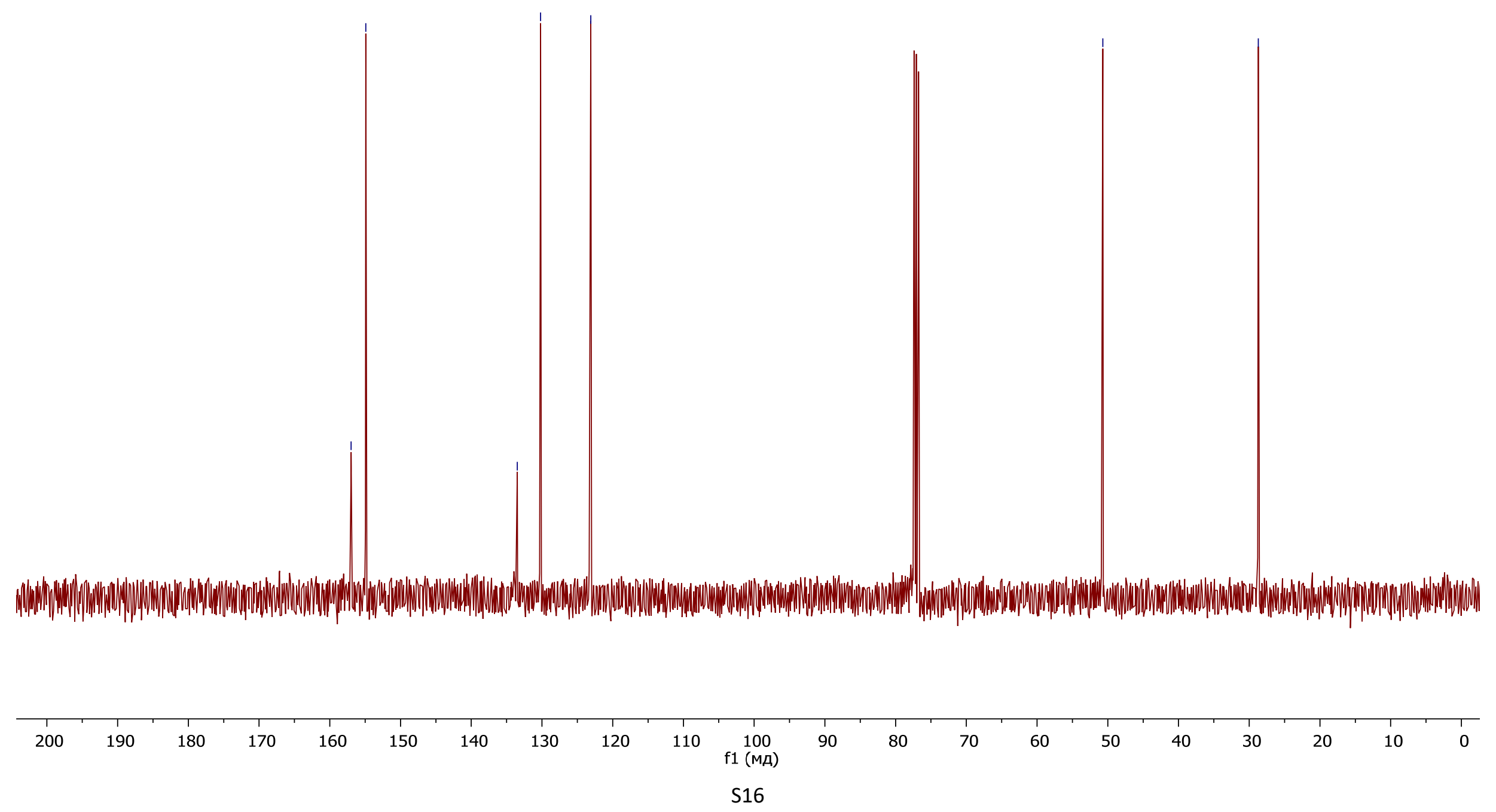


5H,7H,8H-pyrano[4,3-b]pyridine (23I), ${ }^{1} \mathrm{H}$ NMR (500 MHz, $\mathrm{CDCl}_{3}$ )

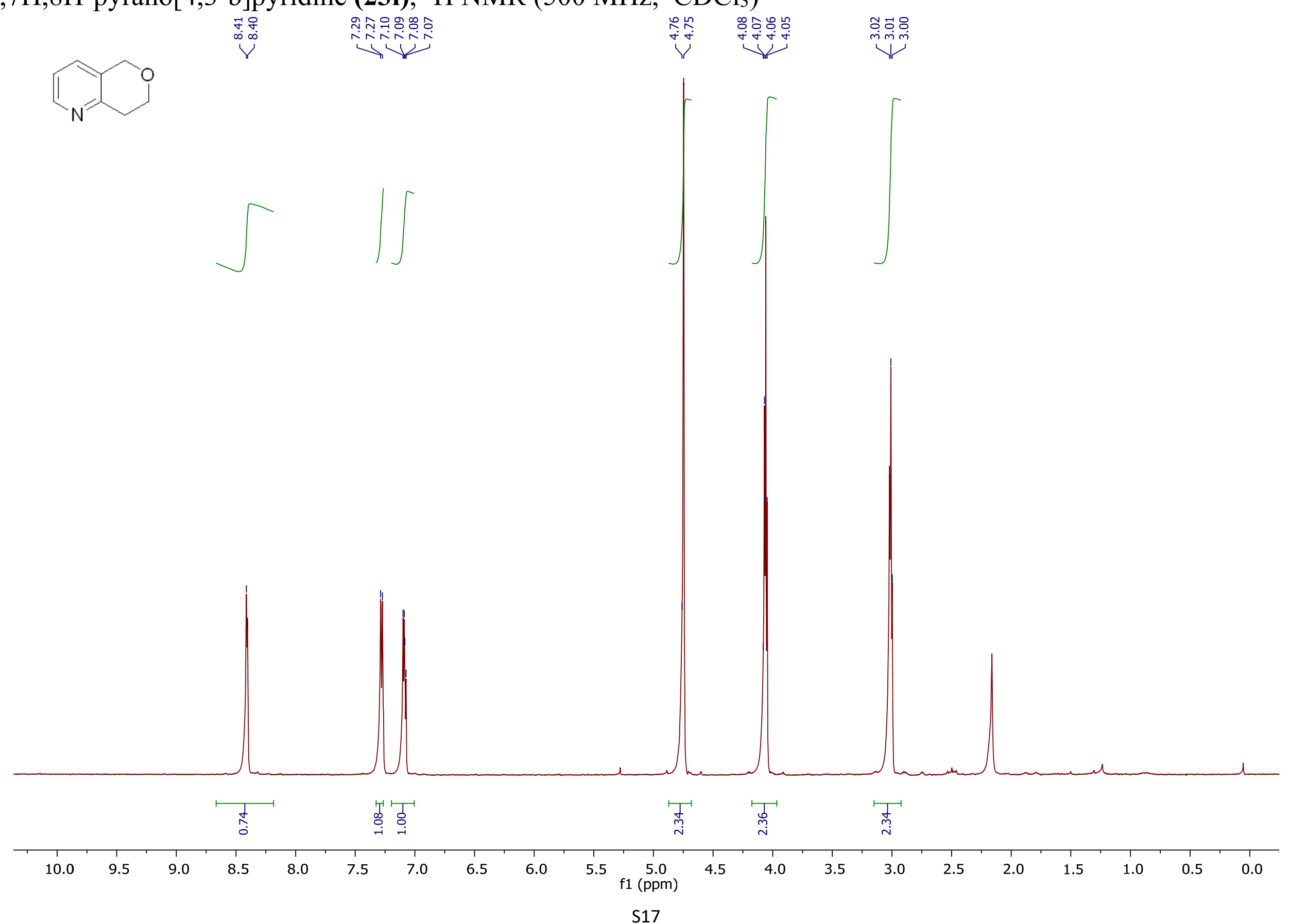


5H,7H,8H-pyrano[4,3-b]pyridine (23I), ${ }^{13} \mathrm{C}\left\{{ }^{1} \mathrm{H}\right\}$ NMR $\left(126 \mathrm{MHz}, \mathrm{CDCl}_{3}\right)$

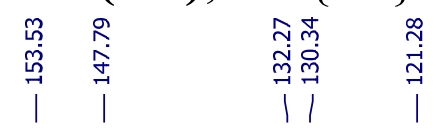
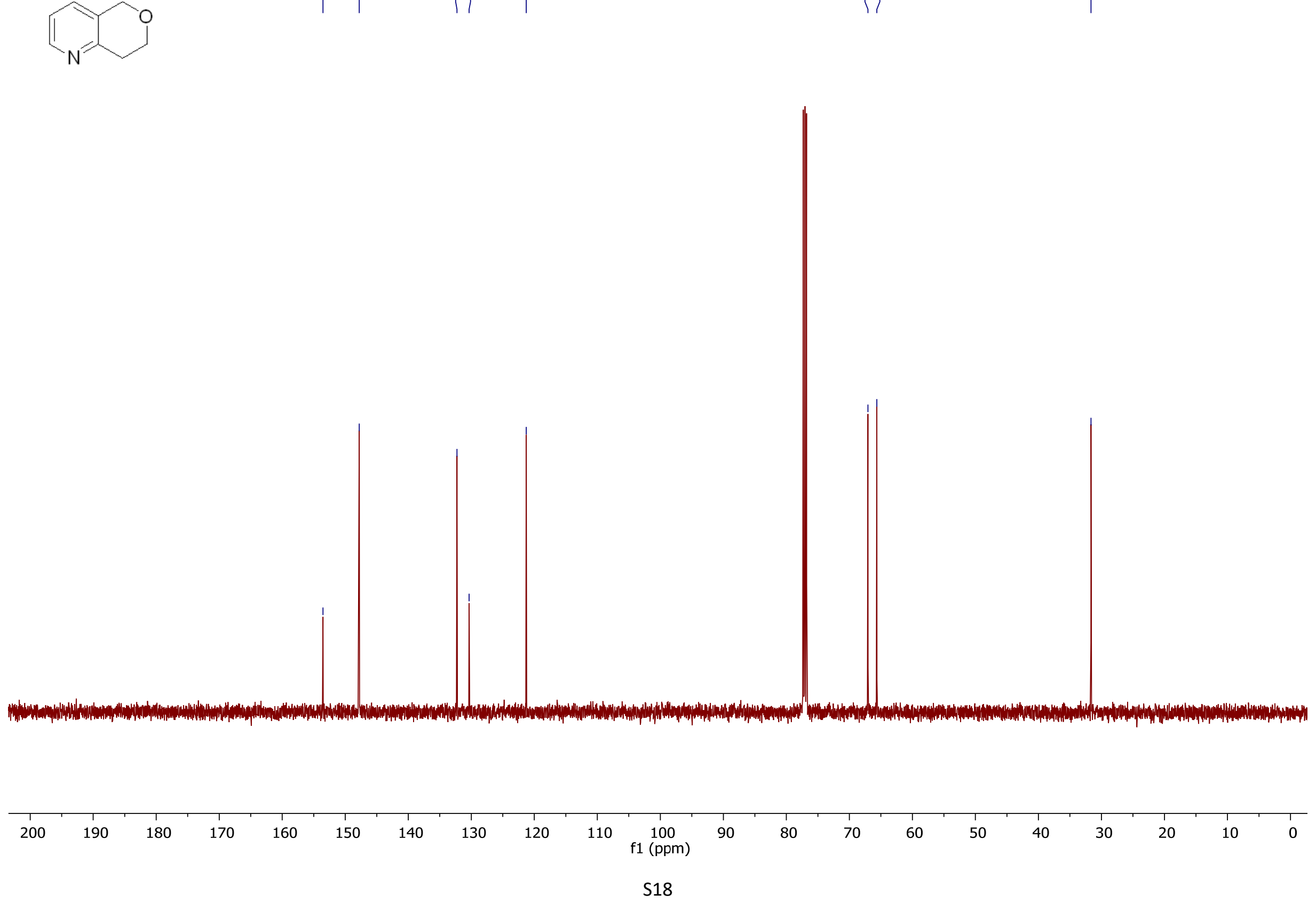
Tert-butyl 5,6,7,8-tetrahydro-1,6-naphthyridine-6-carboxylate (23m), ${ }^{1} \mathrm{H} \mathrm{NMR}\left(400 \mathrm{MHz}, \mathrm{CDCl}_{3}\right)$

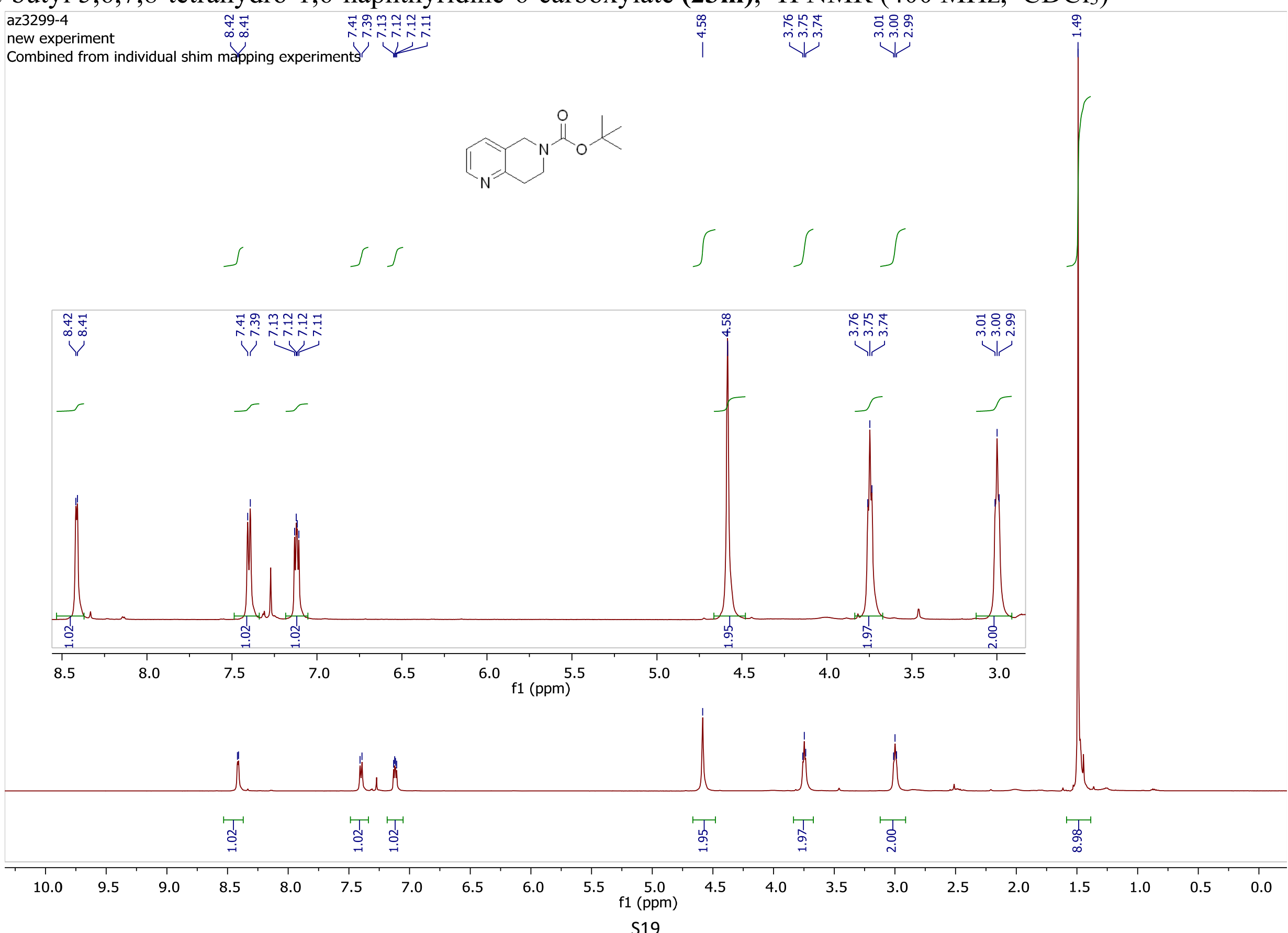


Tert-butyl 5,6,7,8-tetrahydro-1,6-naphthyridine-6-carboxylate (23m), ${ }^{13} \mathrm{C}\left\{{ }^{1} \mathrm{H}\right\} \mathrm{NMR}\left(126 \mathrm{MHz}, \mathrm{CDCl}_{3}\right)$

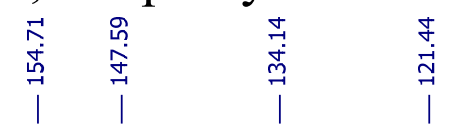

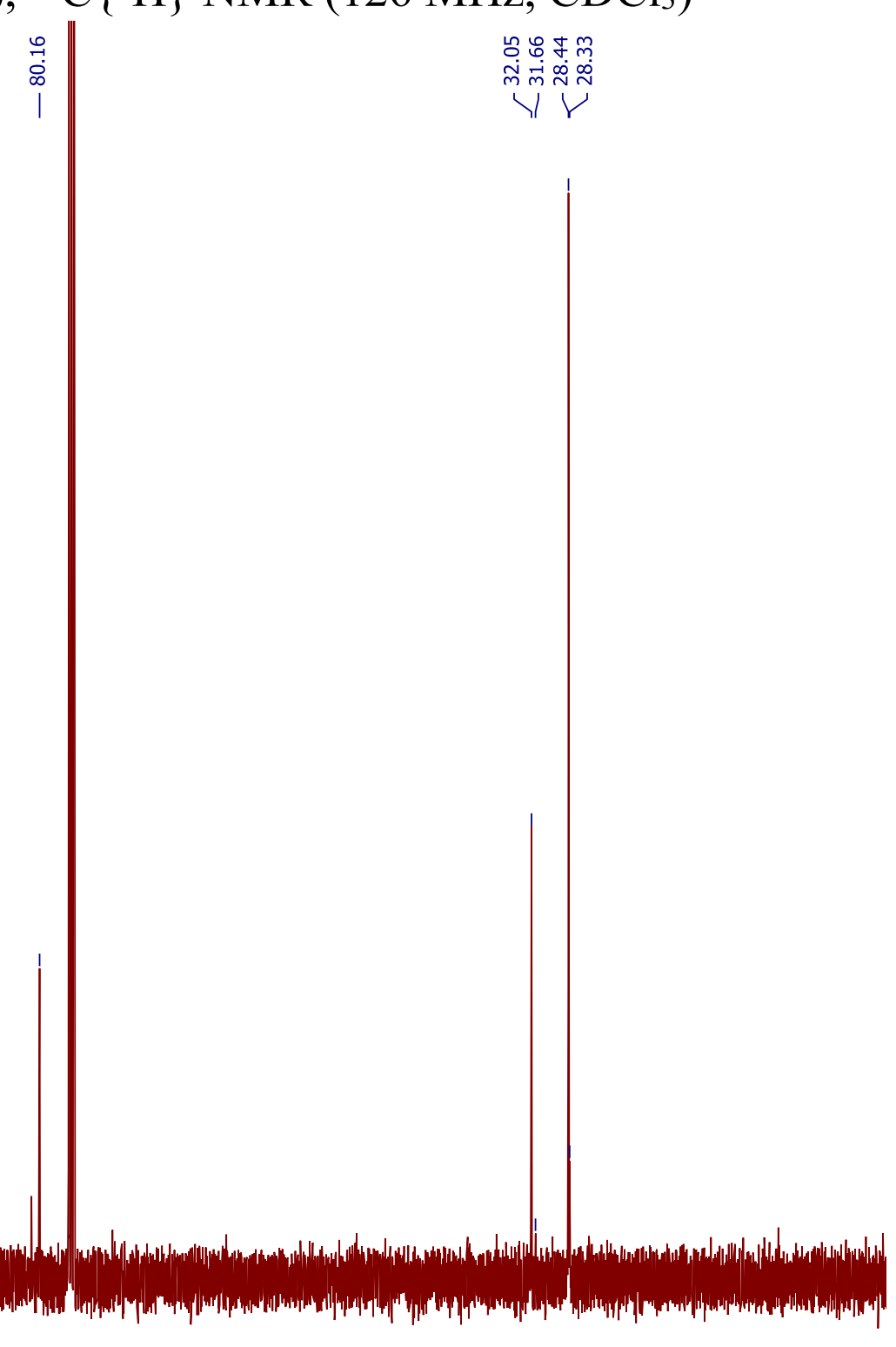


Tert-butyl 1,2,3,4-tetrahydro-1,5-naphthyridine-1-carboxylate (23p), ${ }^{1} \mathrm{H}$ NMR $\left(400 \mathrm{MHz}, \mathrm{CDCl}_{3}\right)$

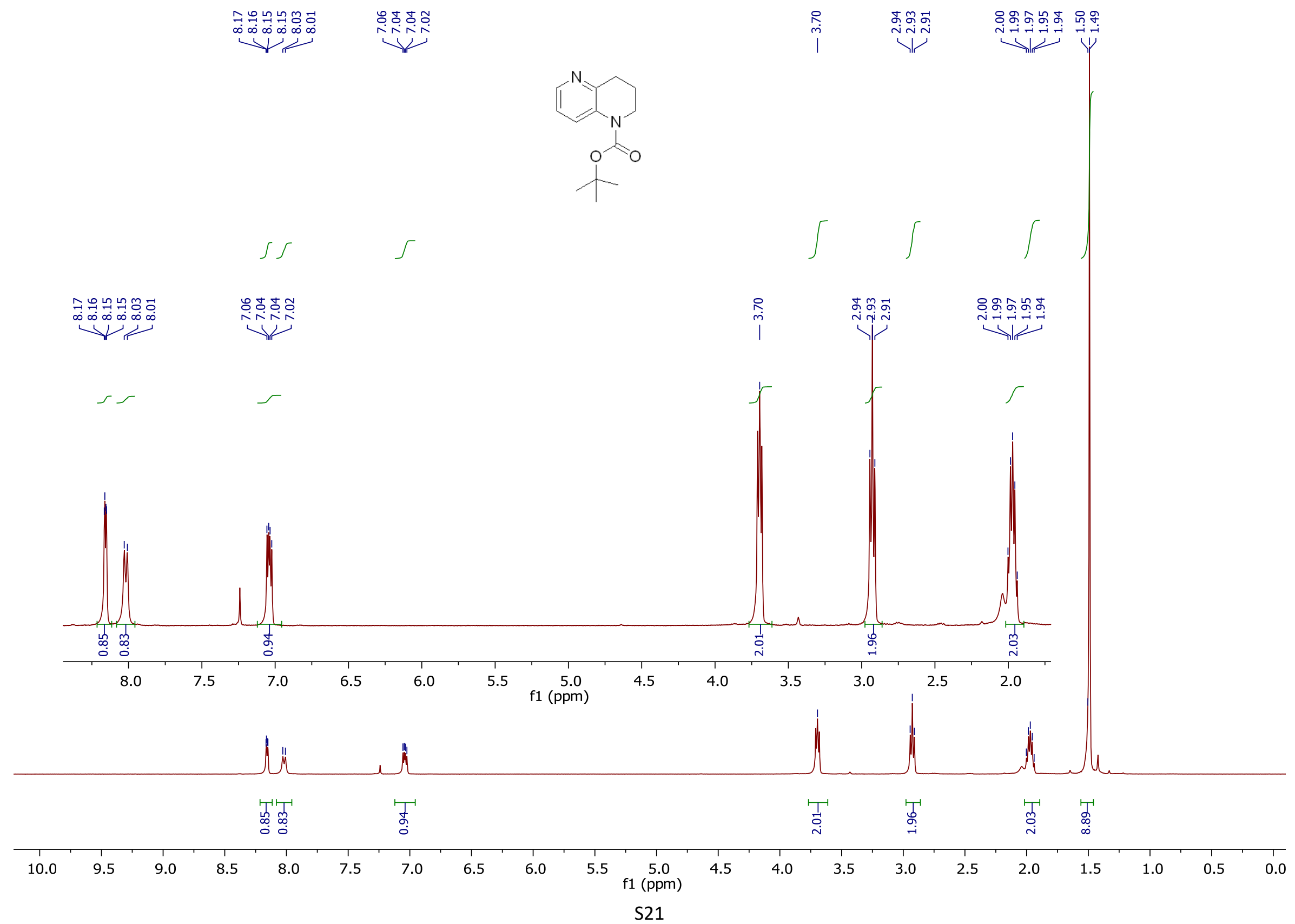


Tert-butyl 1,2,3,4-tetrahydro-1,5-naphthyridine-1-carboxylate (23p), ${ }^{13} \mathrm{C}\left\{{ }^{1} \mathrm{H}\right\} \mathrm{NMR}\left(101 \mathrm{MHz}, \mathrm{CDCl}_{3}\right)$

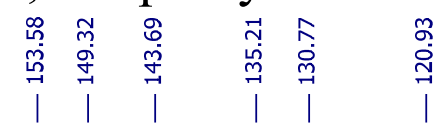

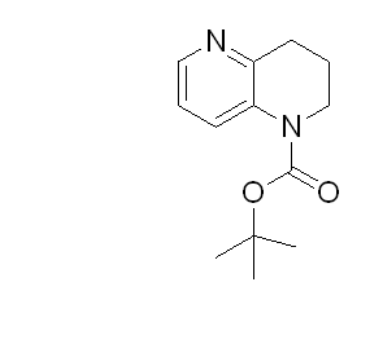

(1)

(2)
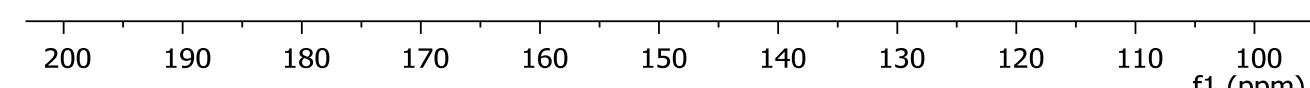

100
$f 1(p p m)$

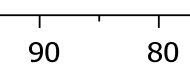


Tert-butyl N-(5,6,7,8-tetrahydroquinolin-6-yl)carbamate (23q), ${ }^{1} \mathrm{H}$ NMR (400 MHz, $\left.\mathrm{CDCl}_{3}\right)$

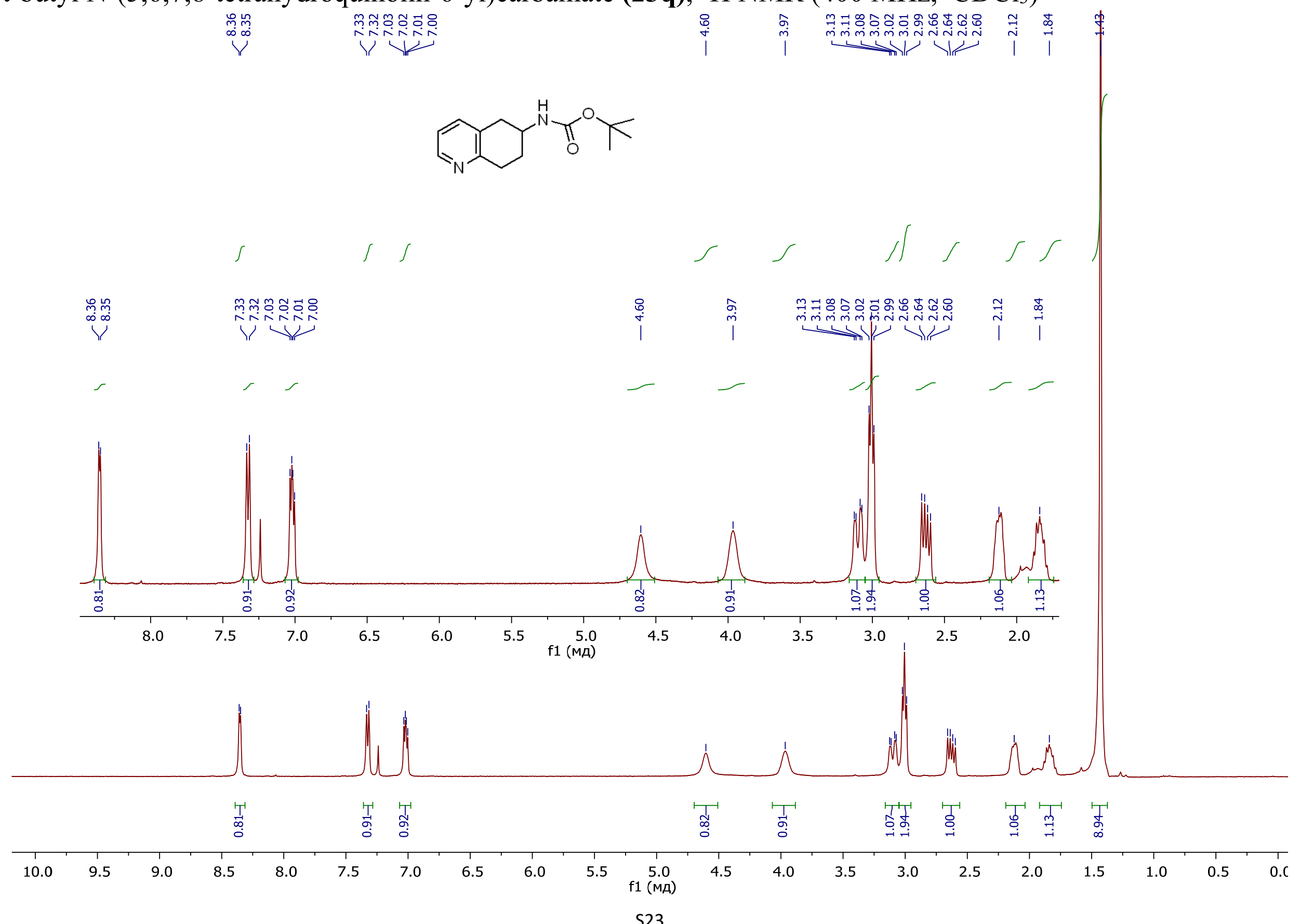


Tert-butyl N-(5,6,7,8-tetrahydroquinolin-6-yl)carbamate (23q), ${ }^{13} \mathrm{C}\left\{{ }^{1} \mathrm{H}\right\} \mathrm{NMR}\left(101 \mathrm{MHz}, \mathrm{CDCl}_{3}\right)$
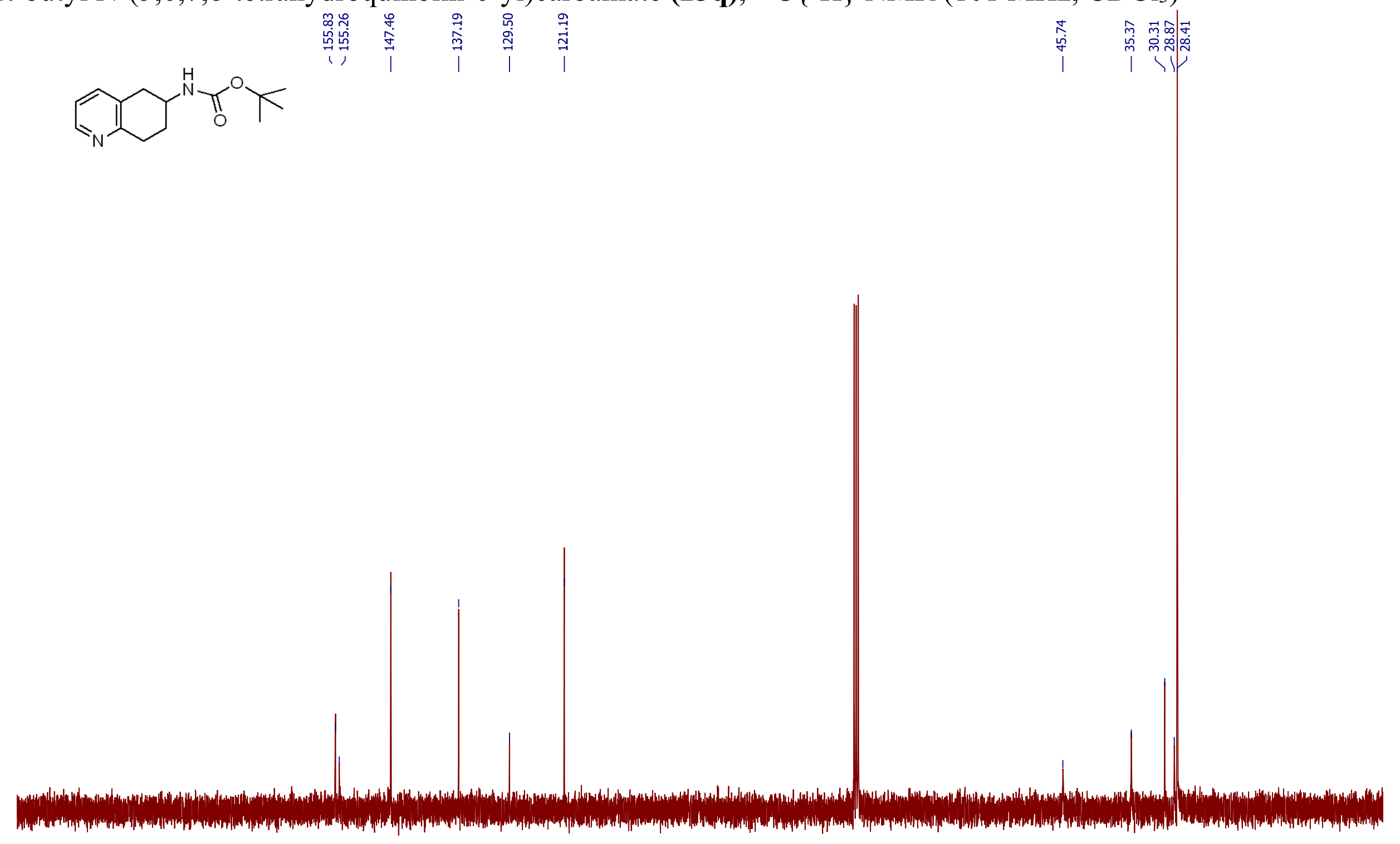

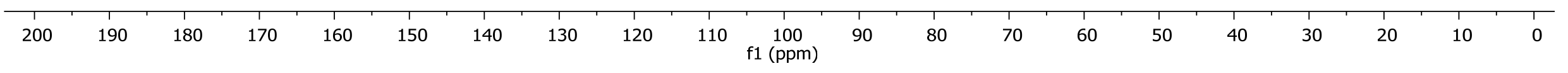


7',8'-dihydro-5'H-spiro[1,3-dioxolane-2,6'-quinoline] (23r), ${ }^{1} \mathrm{H}$ NMR (500 MHz, $\left.\mathrm{CDCl}_{3}\right)$

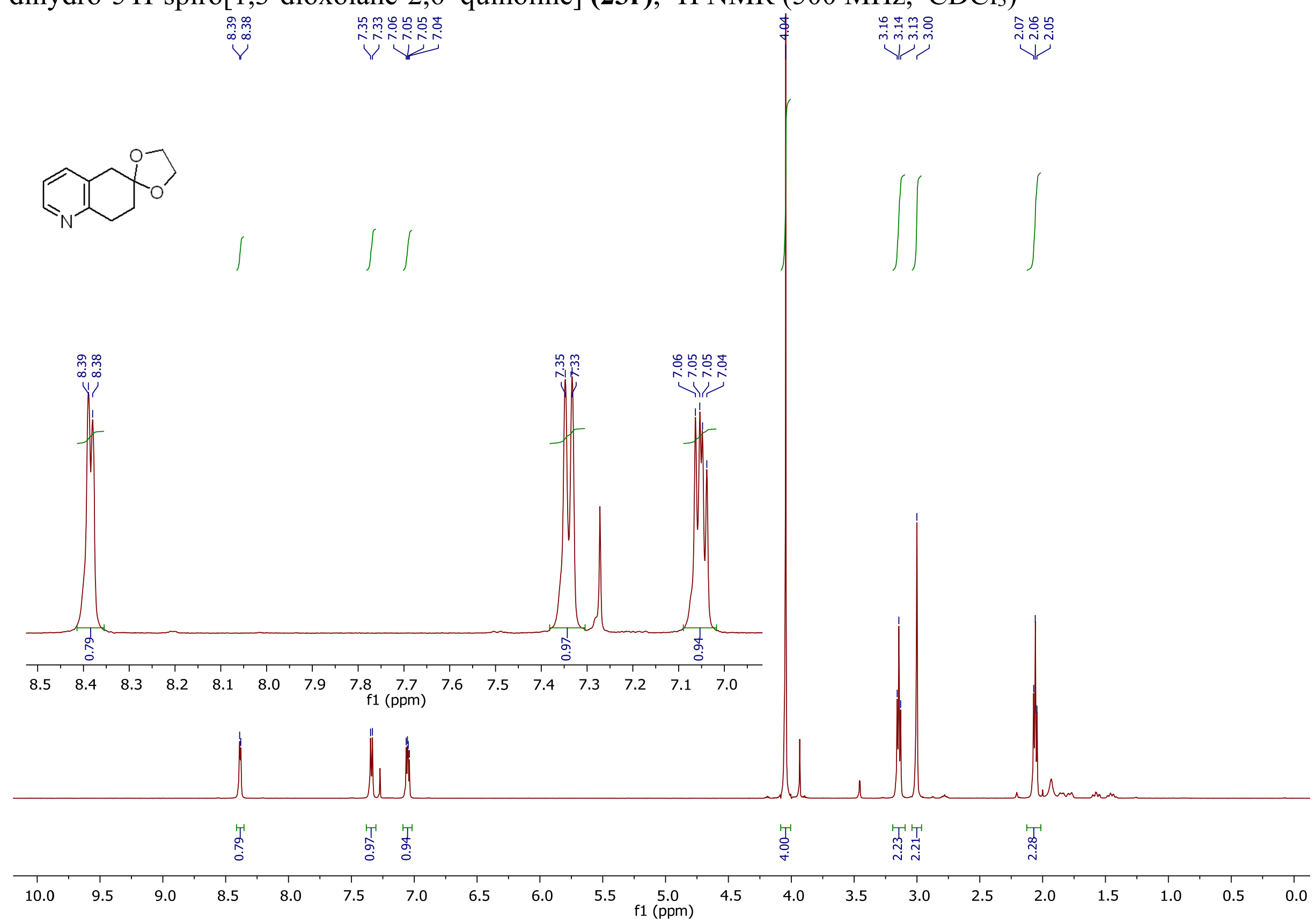


7',8'-dihydro-5'H-spiro[1,3-dioxolane-2,6'-quinoline] (23r), ${ }^{13} \mathrm{C}\left\{{ }^{1} \mathrm{H}\right\} \mathrm{NMR}\left(126 \mathrm{MHz}, \mathrm{CDCl}_{3}\right)$

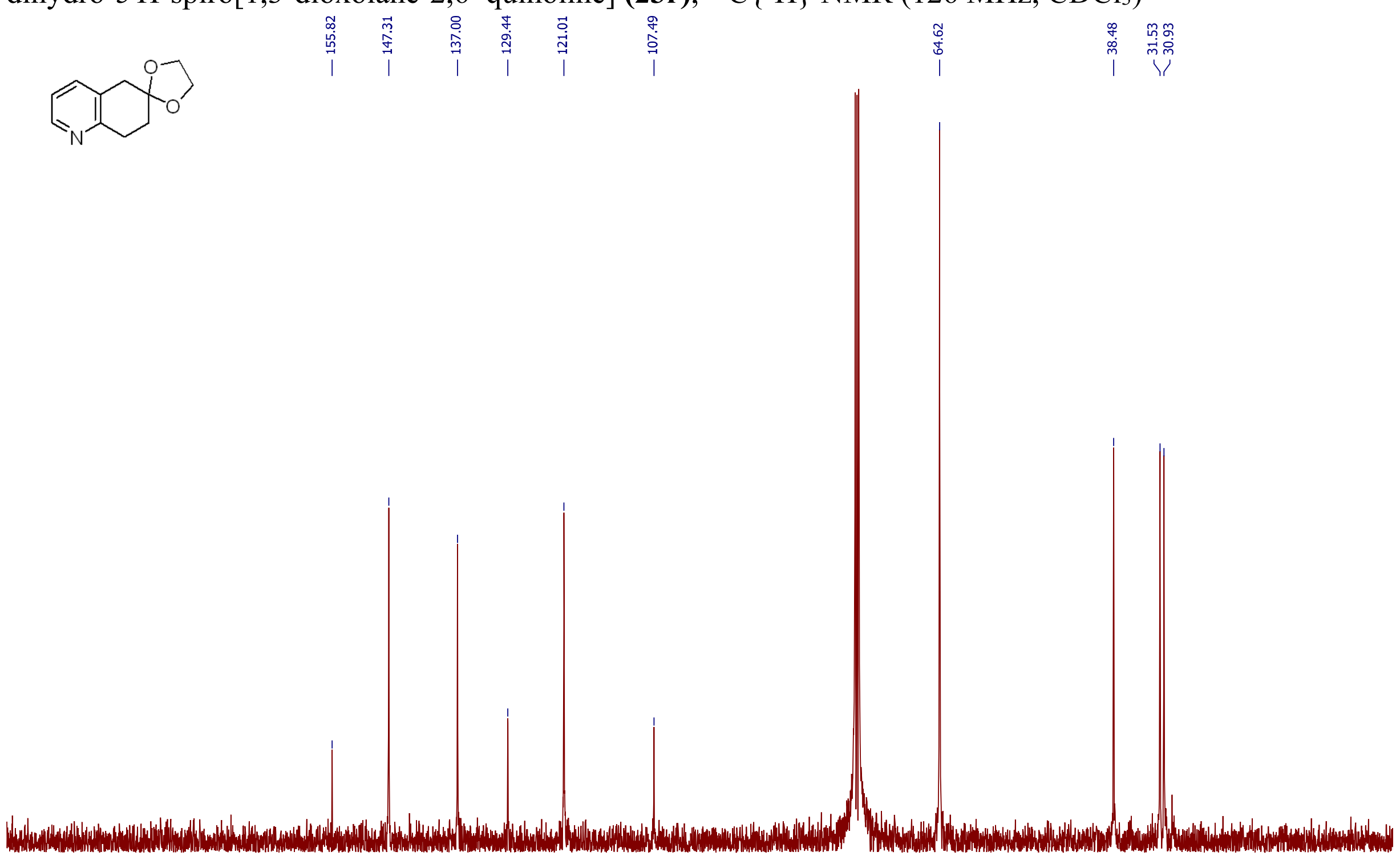


5,6,7,8-tetrahydroquinolin-5-one (23u), ${ }^{1} \mathrm{H}$ NMR (500 MHz, DMSO-d 6 )

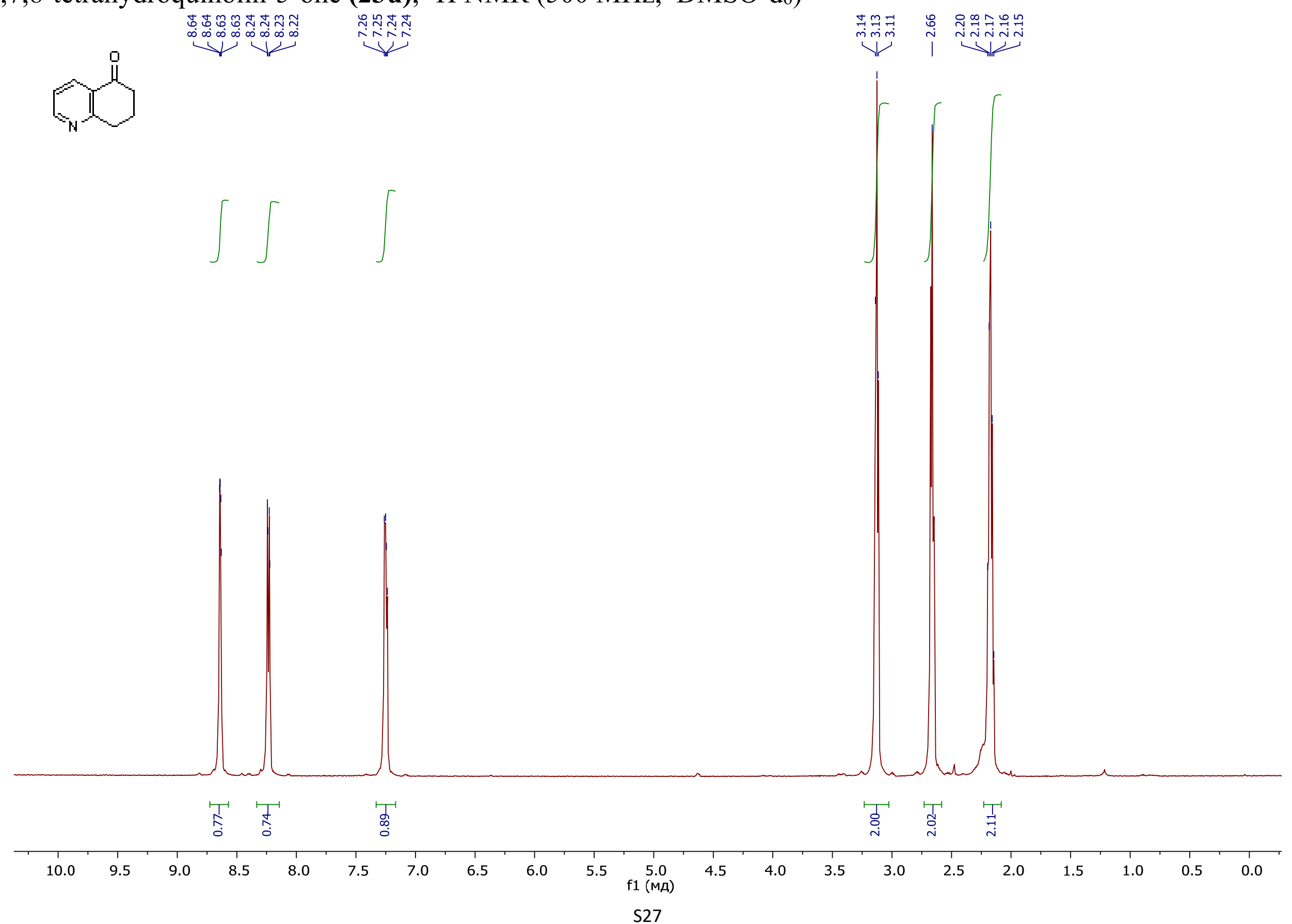


5,6,7,8-tetrahydroquinolin-5-one (23u), ${ }^{13} \mathrm{C}\left\{{ }^{1} \mathrm{H}\right\}$ NMR (126 MHz, DMSO-d 6 )

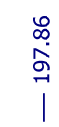

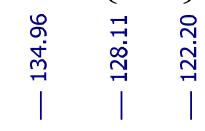<smiles>O=C1CCCc2ncccc21</smiles>

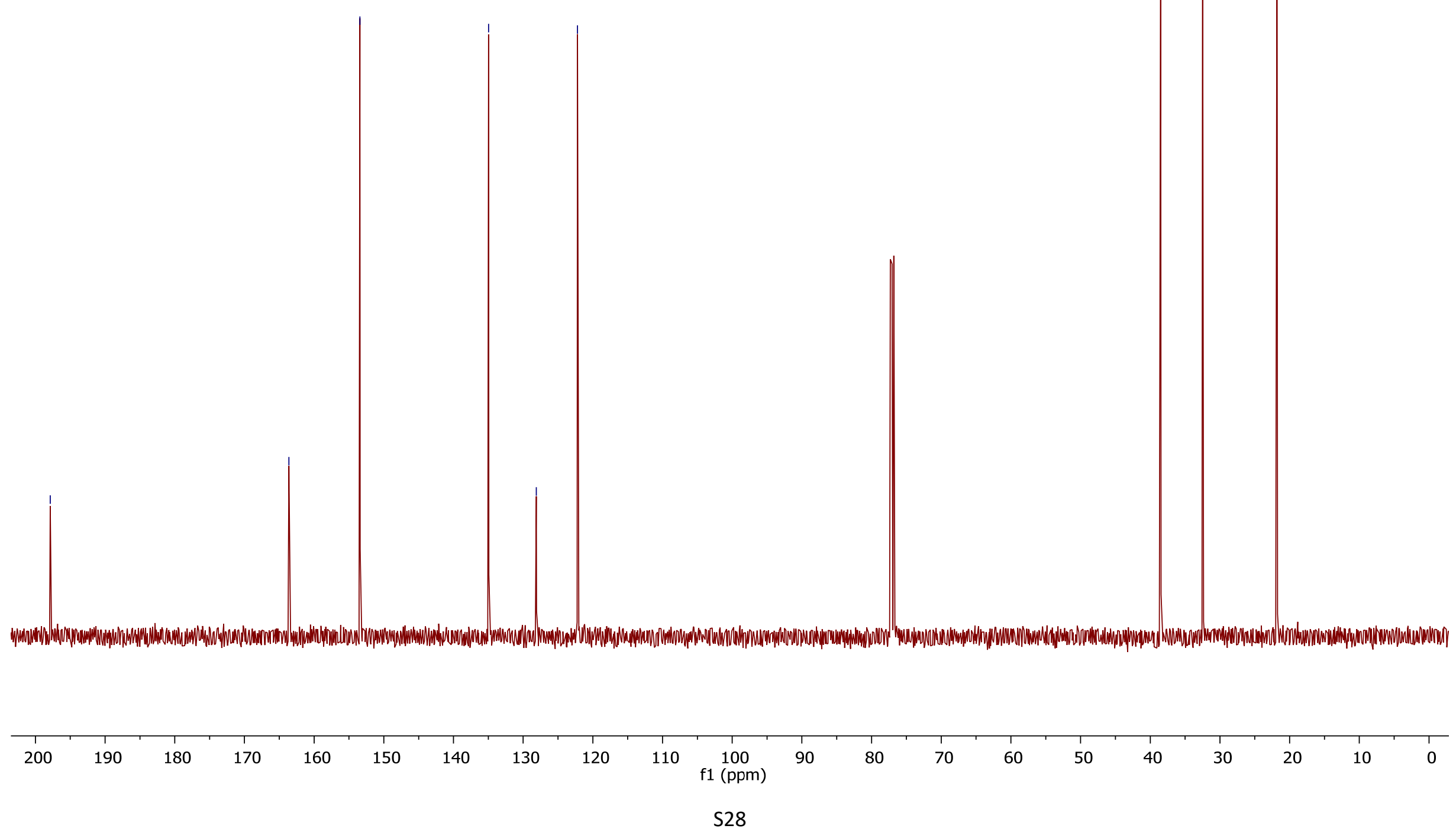


5,6,7,8-tetrahydroquinoline-6-carboxylic acid (24), ${ }^{1} \mathrm{H}$ NMR (400 MHz, DMSO-d 6 )

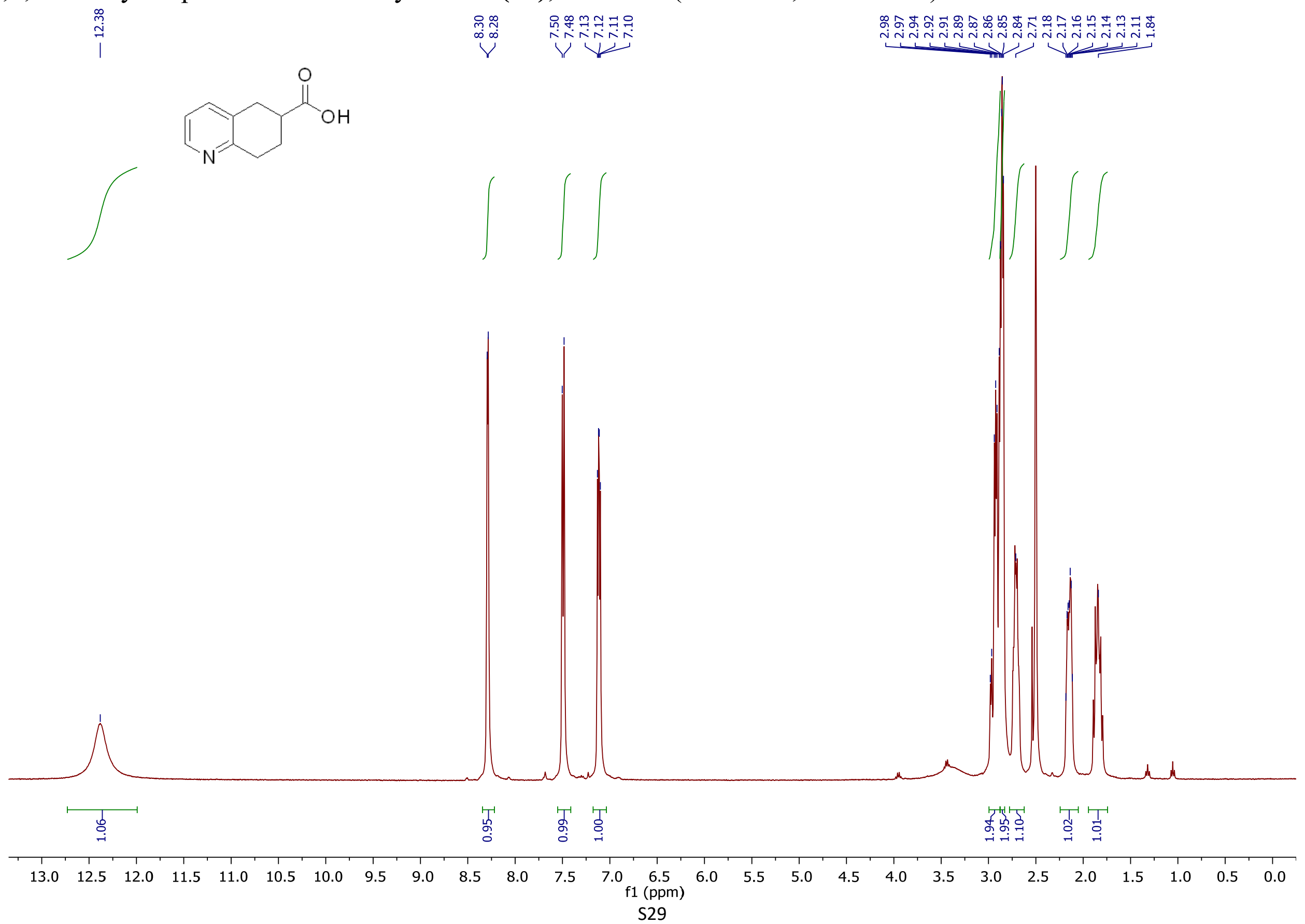


5,6,7,8-tetrahydroquinoline-6-carboxylic acid (24), ${ }^{13} \mathrm{C}\left\{{ }^{1} \mathrm{H}\right\}$ NMR (151 MHz, DMSO-d 6$)$

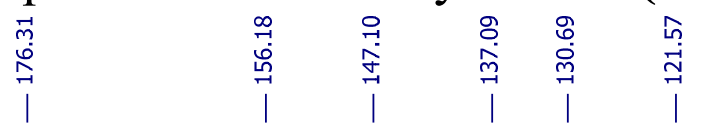
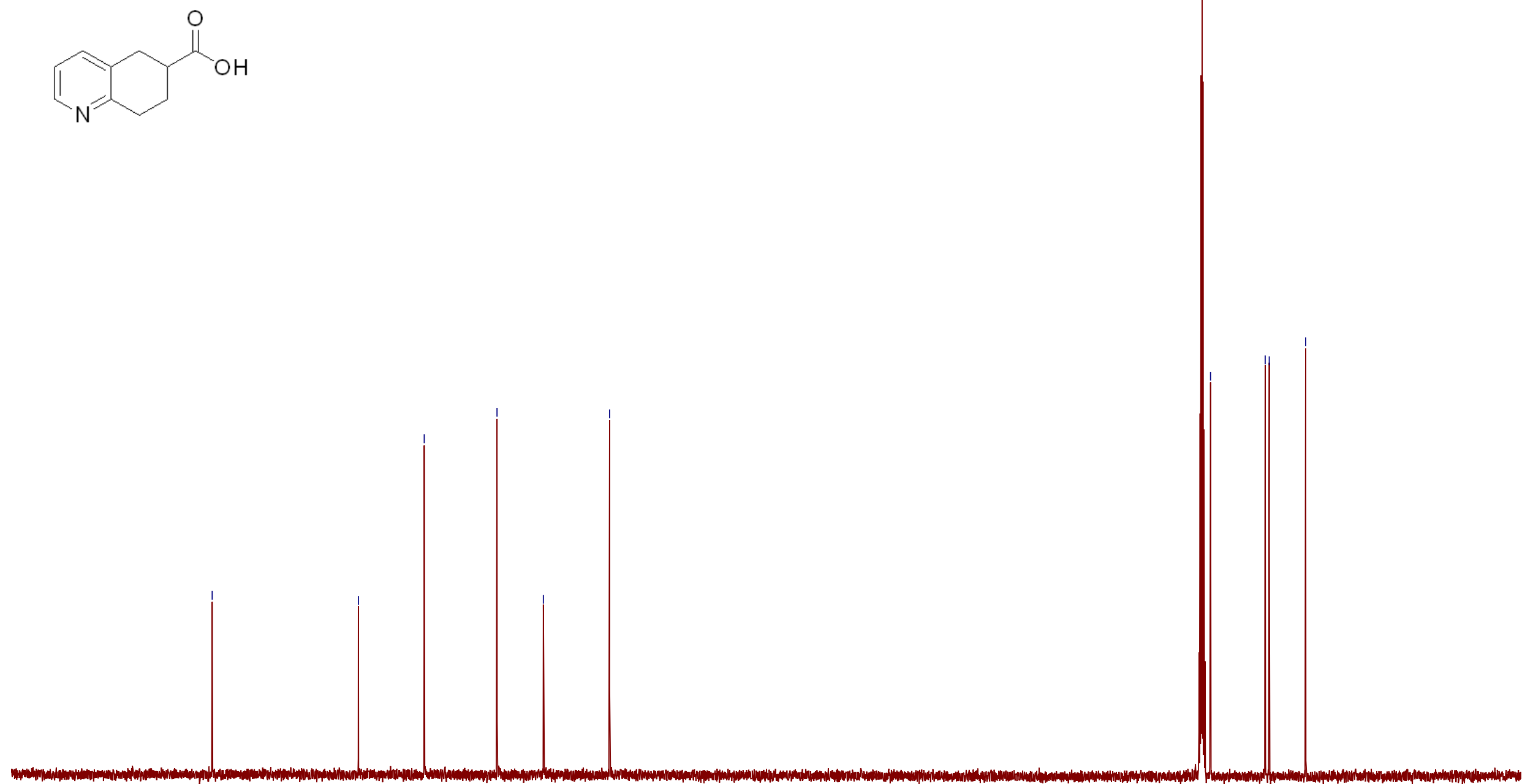
5,6,7,8-tetrahydro-1,6-naphthyridine-1,6-diium chloride (25), ${ }^{1} \mathrm{H}$ NMR (400 MHz, DMSO-d 6 )
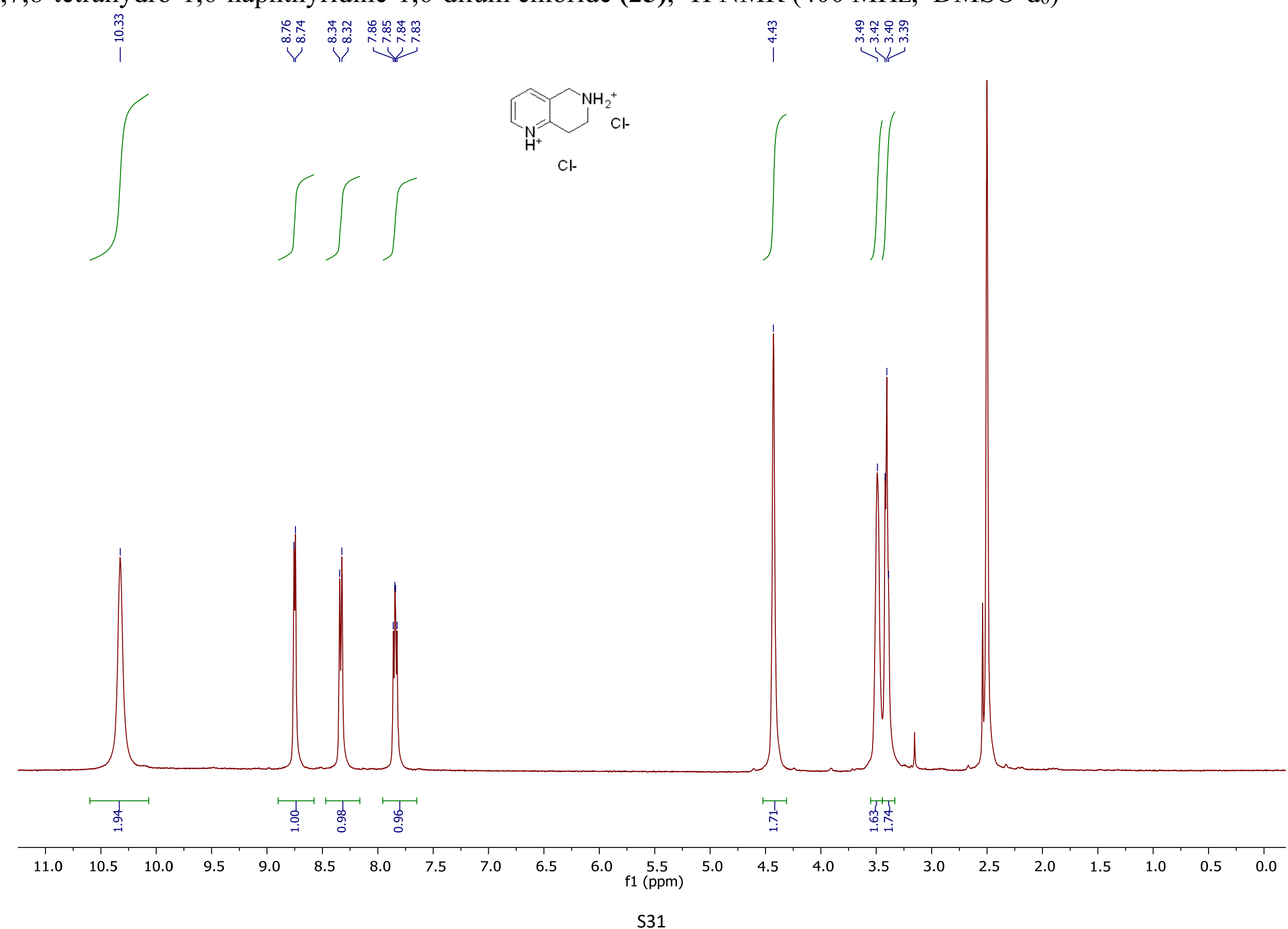
5,6,7,8-tetrahydro-1,6-naphthyridine-1,6-diium chloride (25), ${ }^{13} \mathrm{C}\left\{{ }^{1} \mathrm{H}\right\}$ NMR (151 MHz, DMSO-d $)$

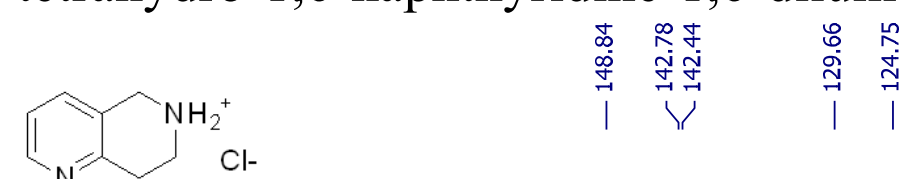

$\mathrm{Cl}-$

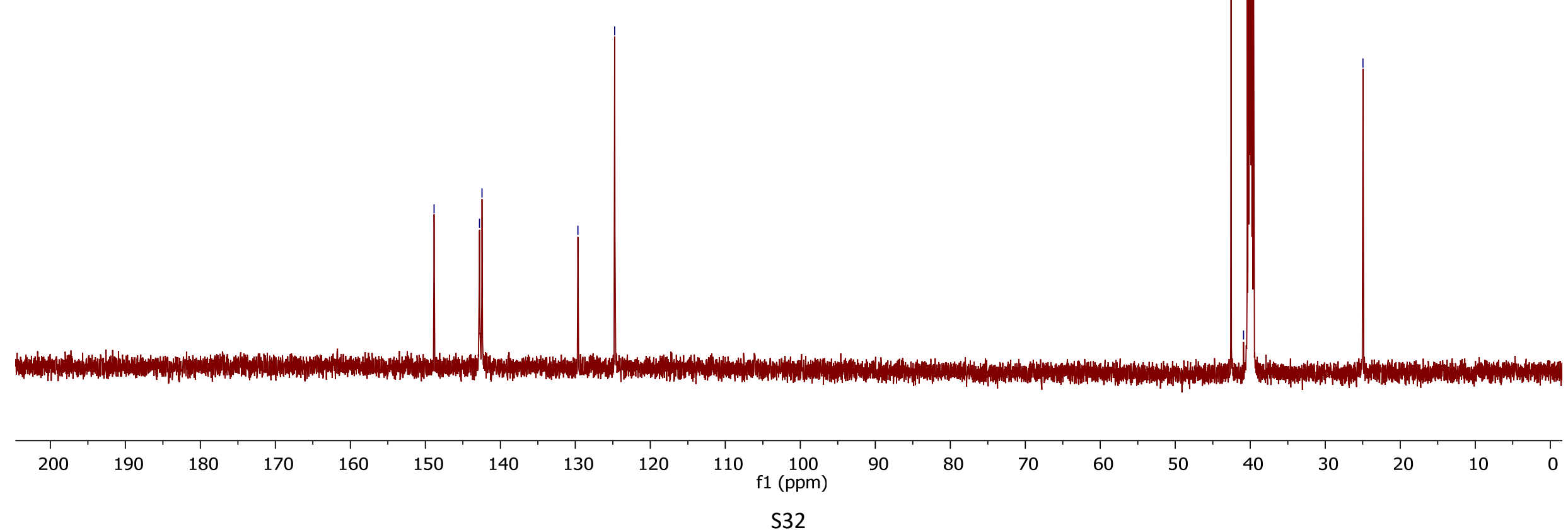


1,2,3,4-tetrahydro-1,5-naphthyridine (26), ${ }^{1} \mathrm{H} \mathrm{NMR}\left(400 \mathrm{MHz}, \mathrm{CDCl}_{3}\right.$ )

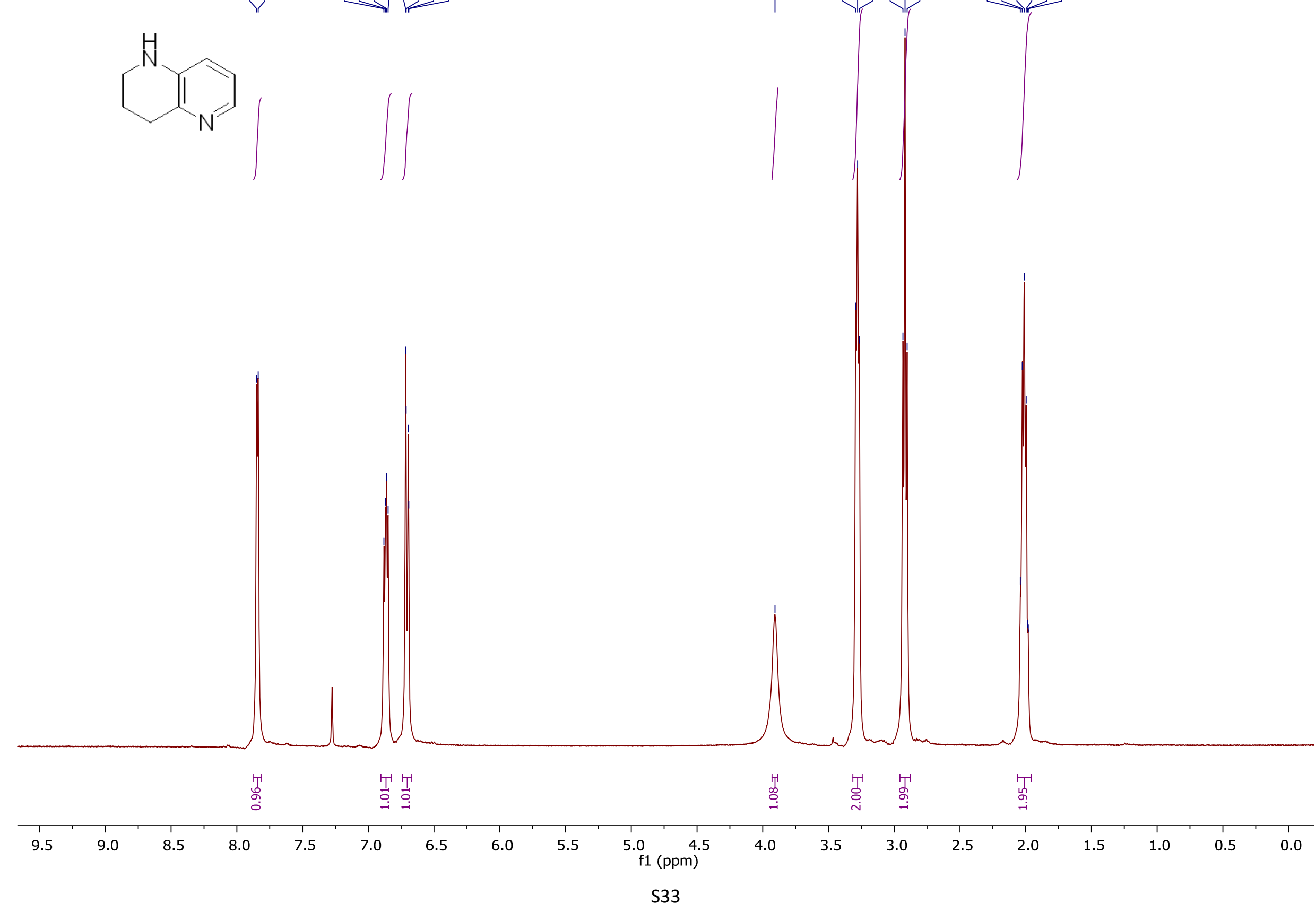

(1) 
1,2,3,4-tetrahydro-1,5-naphthyridine (26), ${ }^{13} \mathrm{C}\left\{{ }^{1} \mathrm{H}\right\} \mathrm{NMR}\left(151 \mathrm{MHz}, \mathrm{CDCl}_{3}\right)$ R1474790_C13<smiles>c1cnc2c(c1)NCCC2</smiles>

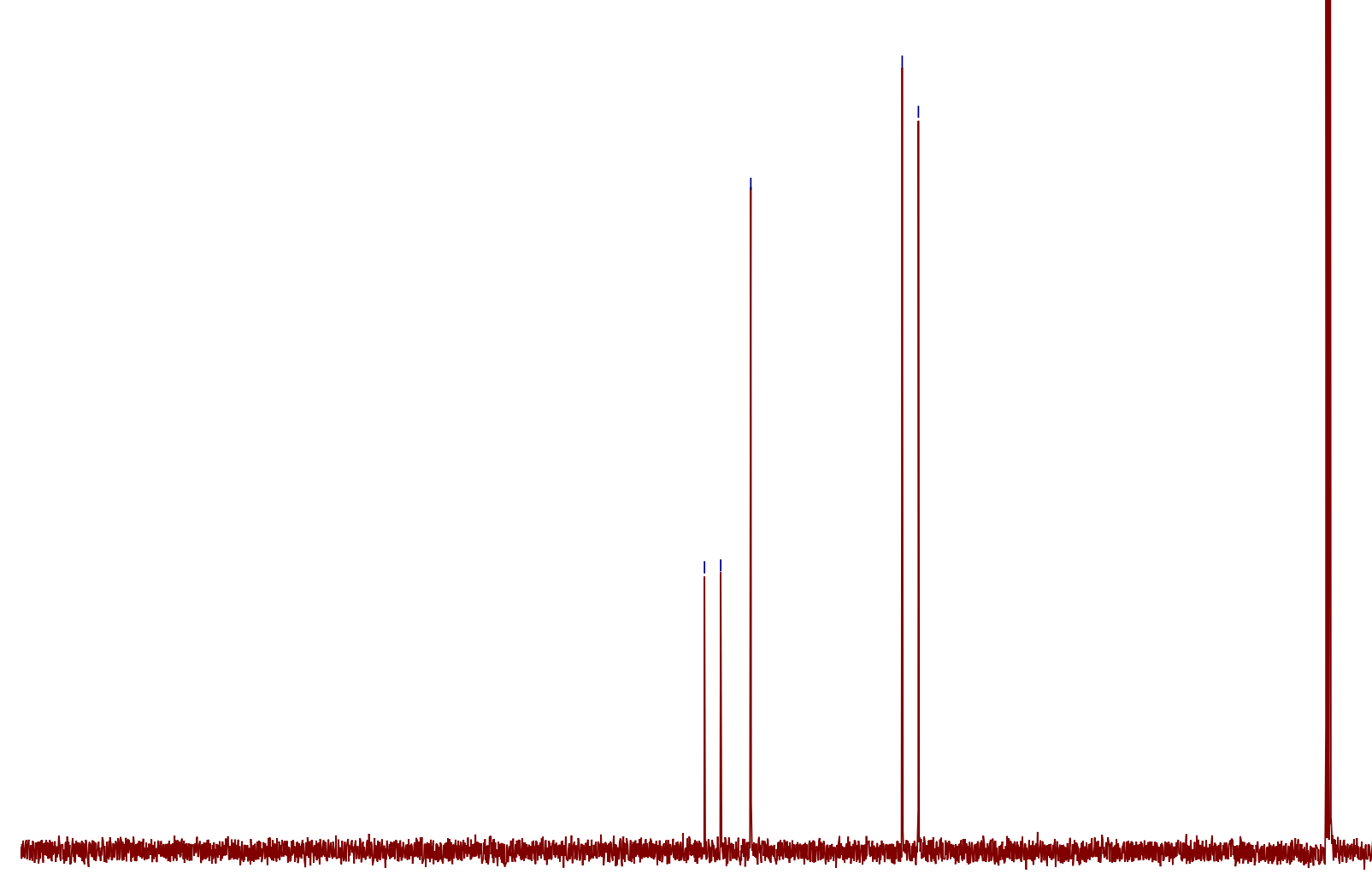


5,6,7,8-tetrahydroquinolin-6-aminium chloride (27), ${ }^{1} \mathrm{H}$ NMR (400 MHz, DMSO-d 6 )

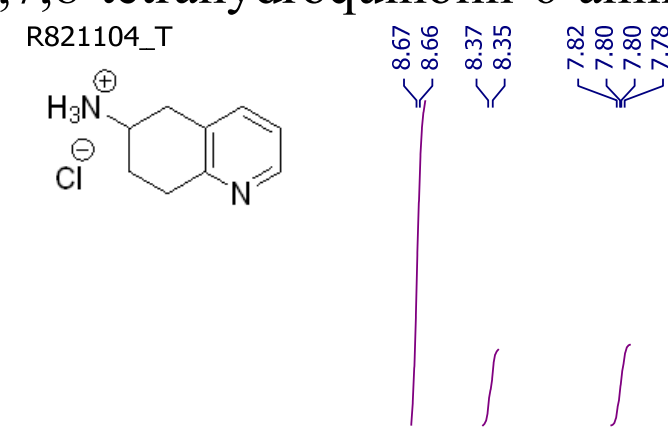

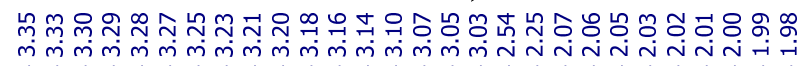

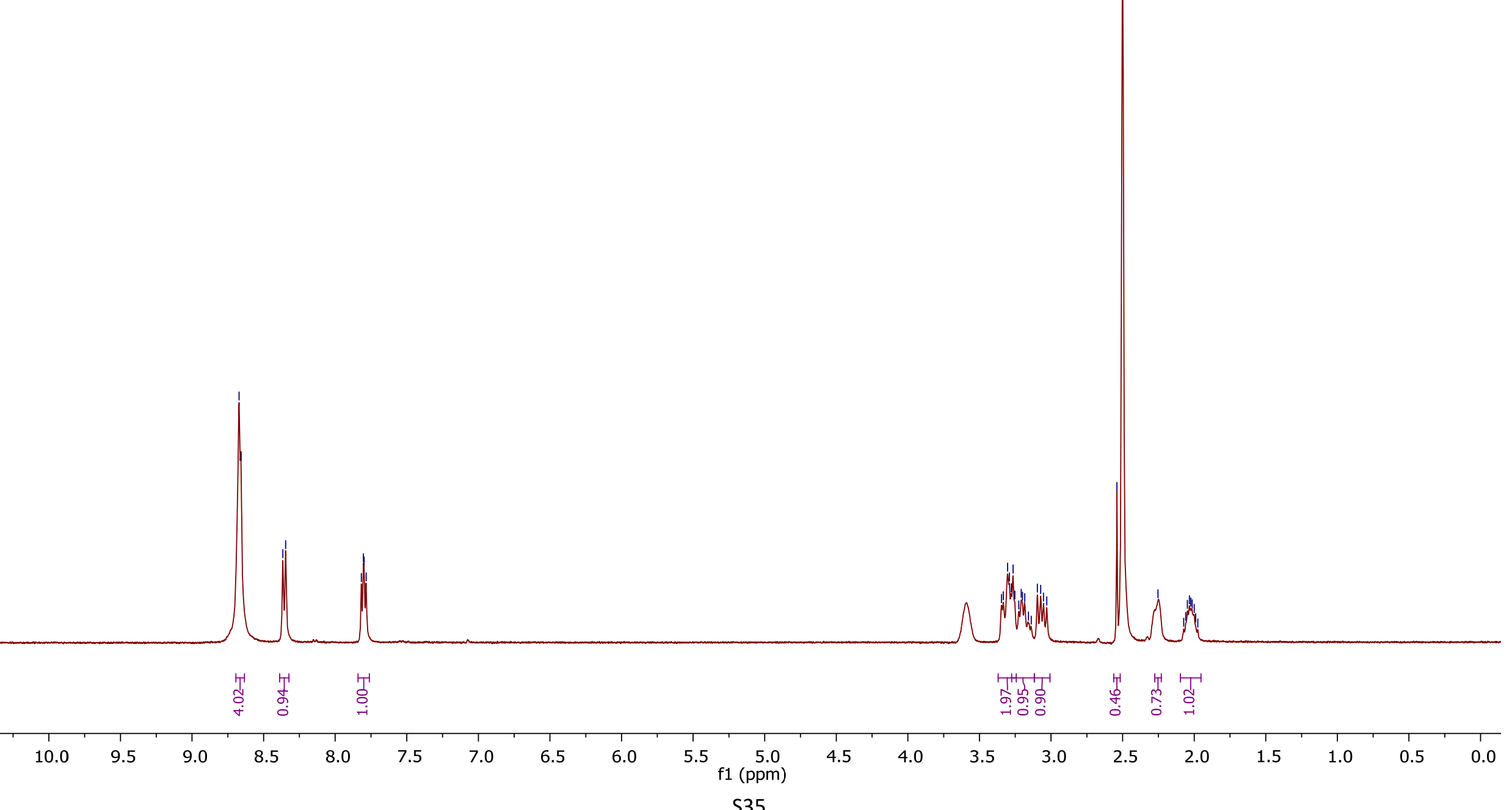


5,6,7,8-tetrahydroquinolin-6-aminium chloride (27), ${ }^{13} \mathrm{C}\left\{{ }^{1} \mathrm{H}\right\}$ NMR (101 MHz, DMSO-d $)$ R821104_C13_T.1.fid

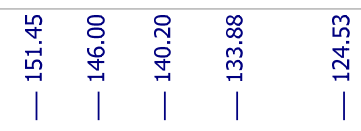<smiles>[NH3+]C1CCc2ncccc2C1</smiles>

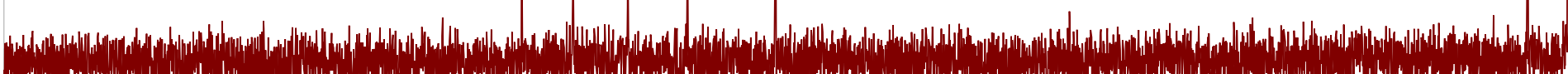
W

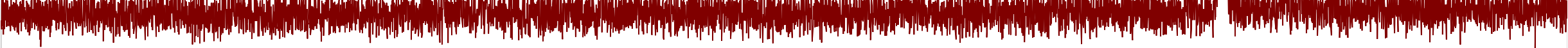

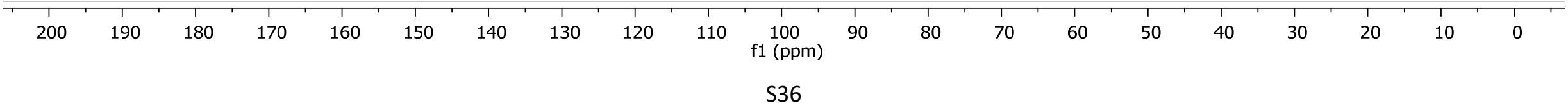


5,6,7,8-tetrahydroquinolin-6-one (28), ${ }^{1} \mathrm{H}$ NMR (400 MHz, $\mathrm{CDCl}_{3}$ )

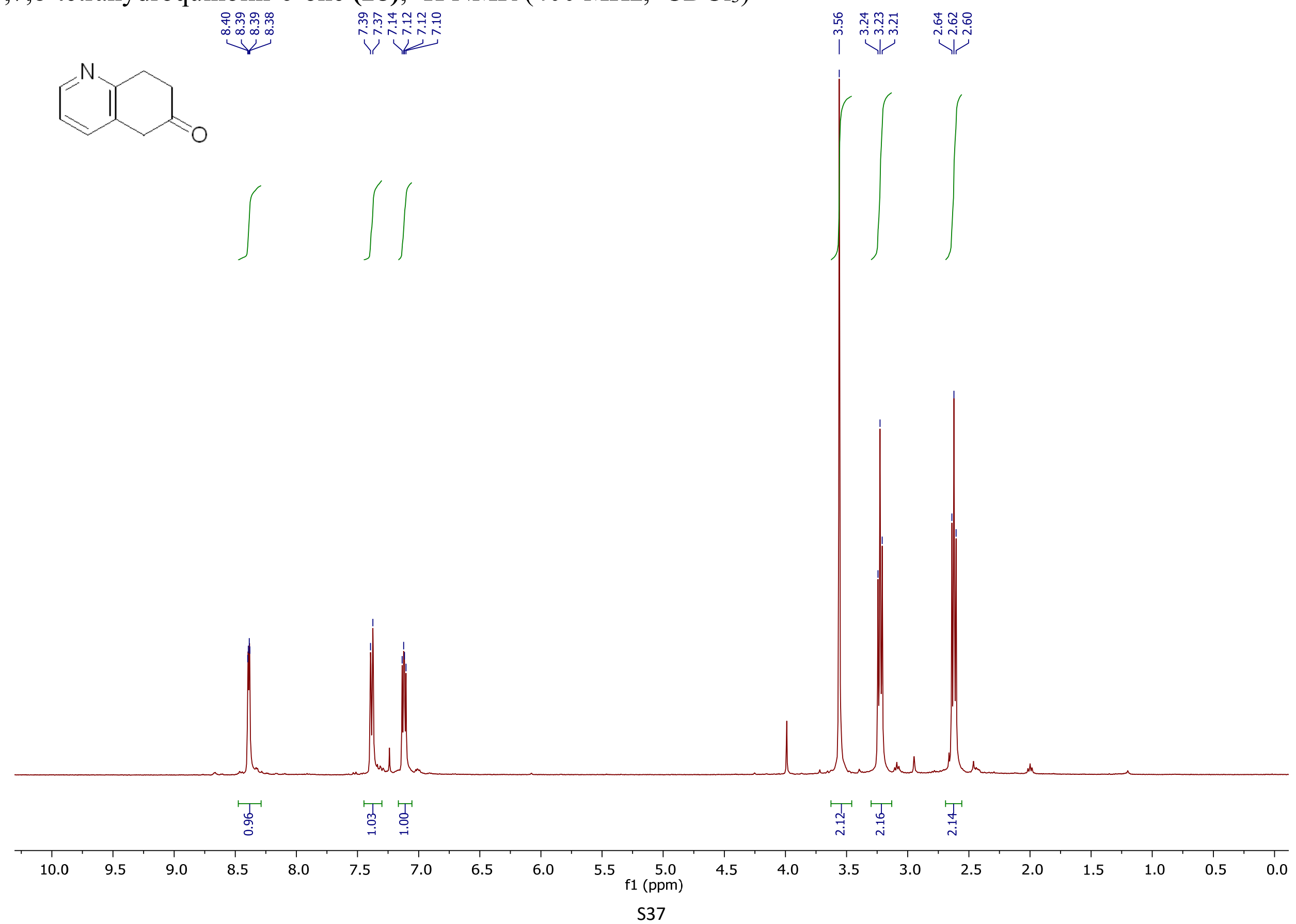


5,6,7,8-tetrahydroquinolin-6-one (28), ${ }^{13} \mathrm{C}\left\{{ }^{1} \mathrm{H}\right\}$ NMR (126 MHz, $\mathrm{CDCl}_{3}$ )

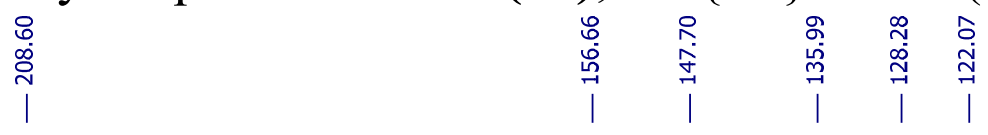

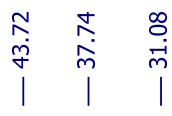<smiles>O=C1CCc2ncccc2C1</smiles>

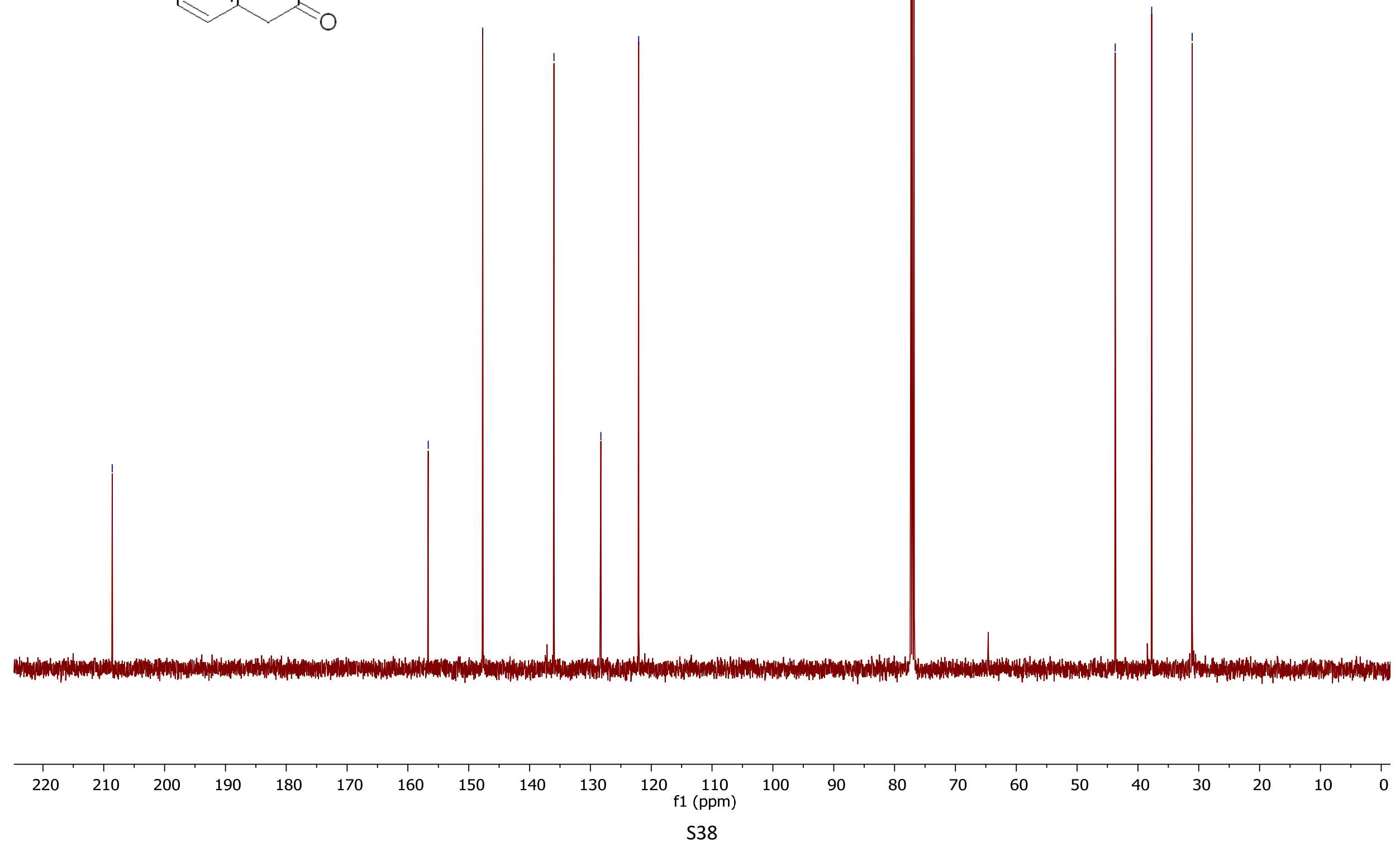

